\title{
Electro-Thermal Analysis of Multi-Fin Devices
}

\author{
Brian Swahn, Member, IEEE, and Soha Hassoun, Senior Member, IEEE
}

\begin{abstract}
As device dimensions shrink into the nanometer range, power and performance constraints prohibit the longevity of traditional MOS devices in circuit design. FinFETs, a quasi-planar double-gated device, has emerged as a replacement. While finFETs provide promising electrostatic characteristics, they have the potential to suffer from significant self heating. We study in this paper self heating in multi-fin devices. We first develop thermal models for an individual fin with flared channel extensions and for multi-fin devices. We analyze several fin geometric parameters (fin width, and (gate) length) and investigate how fin spacing, fin height, gate oxide thickness and gate height affect the maximum fin temperatures in rectangular and flared channel extensions. Our data derived from numerical simulation validates our findings. We develop a novel metric, metric for electro-thermal sensitivity (METS), for measuring device thermal robustness. We use the metric to investigate electro-thermal device sensitivities. The metric, while applied to finFETs in this paper, is general and can be applied to any type of device for which coupled electrical and thermal models exist. Our work is the first to address thermal issues within multi-fin devices and to develop a widely-applicable electro-thermal metric.
\end{abstract}

Index Terms-Electro-thermal effects, finFET, integrated circuit (IC) design, thermal analysis, VLSI.

\section{INTRODUCTION}

$\mathbf{N}$ EXT-GENERATION VLSI circuits will be composed of devices with dimensions in the nanometer range (e.g., sub-100-nm gate lengths). For many decades, planar devices have been the favorites for both bulk and SOI processing. Planar devices however are susceptible to scaling effects. Subthreshold conduction (e.g., leakage current) is the major hurdle that these devices have yet to overcome. Leakage current stems from decreased oxide thicknesses, higher substrate dopings, and decreased channel lengths. A lowered threshold voltage to obtain better performance at lower operating voltages further exacerbates the leakage problem.

The 2003 International Technology Roadmap for Semiconductors predicts several transistor improvements, including strained Si-channels, ultra-thin bodies, and metallic junctions [1]. It also predicts the move towards double-gate devices which allow more than one gate terminal to control the transistor channel. Among double-gated devices, the finFET, originally dubbed as the folded-channel MOSFET [2], promises better alignment of the double gates. Moreover, finFETs have high current drive and offer substantially better control over leakage and short channel effects. Like a traditional MOSFET,

Manuscript received January 29, 2007; revised June 11, 2007 and July 30, 2007. This work was supported by NSF grant CCF-0429921, and a gift from Altera Corporation.

B. Swahn is with Analog Device, Inc., Wilmington, MA 01887 USA (e-mail: brian@swahnsoft.com).

S. Hassoun are with the Department of Computer Science, Tufts University, Medford, MA 02451 USA (e-mail: swahn@ee.tufts.edu).

Digital Object Identifier 10.1109/TVLSI.2008.2000455

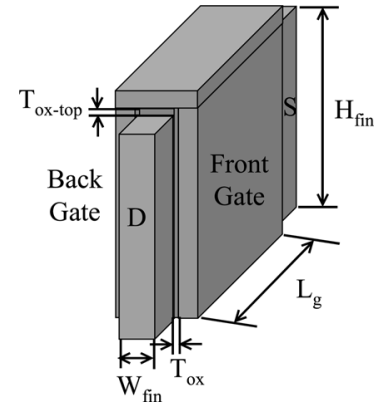

Fig. 1. FinFET device.

the finFET is composed of a channel, a source, a drain, and a gate. The channel is embodied in a fin protruding out of the wafer plane. The fin is fabricated out of either undoped or lightly doped silicon. The gates of the finFET are created by wrapping the gate material around the three sides of the silicon fin, resulting in self-aligned front and back gates. Fig. 1 shows the geometric parameters for a finFET. $L_{g}$ is the gate length; $H_{\text {fin }}$ is the fin height; $W_{\text {fin }}$ is the fin width or thickness; $t_{\mathrm{ox}}$ is the oxide thickness between the side gates and the fin; $t_{\mathrm{ox}-\text { top }}$ is the oxide thickness between the top gate and the fin. The width of a finFET is defined as $W=2 \times H_{\text {fin }}$. FinFET fabrication uses a typical planar fabrication process with several new masks introduced into the process flow [3]. Hisamoto et al. [4] devised one of the first finFET fabrication flows, and several others have improved on it [5]-[8]. The main flow roughly consists of etching a fin out of the silicon wafer, depositing the source and drain, depositing the gate oxides, and finally depositing the gate material.

While providing promising electrostatic characteristics, finFETs, along with other nanoscale devices, pose nontrivial selfheating challenges. With the potential impact of temperature on performance [9] and reliability, thermal device design becomes important, specially for analog circuits. We outline finFET selfheating challenges.

- Device thermal modeling has consisted mainly of modeling a device as a transient 3-D heat flow problem [10], [11]. The temperature at any point within the device can be found at any instant in time. The heat diffusion equation however fails to capture the dominant thermal energy transport mechanism due to phonons, particles that transport energy, and atomic lattice vibrations. Recently, the Boltzmann Transport Equation (BTE) was used to estimate the hot spots associated with the drain regions [12], [13]. For example, Sverdrup, Ju, and Goodson compared the BTE to classic heat diffusion temperature estimations within a MOSFET device. They found the heat diffusion equation underestimates the maximum device temperature, when compared to BTE estimates, by as much as $159 \%$ [12]. 


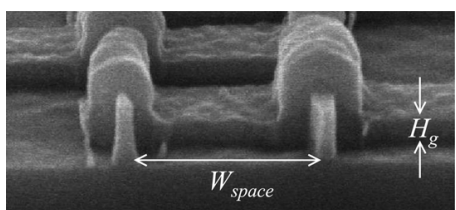

Fig. 2. Multi-fin device [18].

- The small and confined dimensions of the fin reduce the thermal conductivity (which increases the thermal resistance) of the device due to reduced phonon mean path [14]. Heat transport out of the device is hindered and the device temperature rises.

- Heat dissipation is sensitive to the dimensions of source and drain extensions [15]. Careful device thermal analysis is needed to balance the device's electrical characteristics with thermal ones [5].

- SOI finFETs are even more susceptible to self heating than bulk ones: SOI thermal conductivity is two orders of magnitude less than that of silicon [16].

- FinFET thermal problems are further exasperated with the construction of wider finFETs built using parallel fins between the source and the drain areas, as shown in Fig. 2 [17]. These fins are tightly laid out. Heat removal from the middle fins is not as efficient as the removal from the end fins.

Our paper studies the effects of steady-state self-heating in multi-fin devices on performance. Our investigation is based on the ultra-thin body (UTB) SOI thermal model introduced by Pop, Dutton, and Goodson [15]. We first propose a flared channel extension thermal model of each individual fin. We then extend that model to account for multiple fins. By carefully examining the multi-fin model, we are able to identify the key parameters that affect the maximum temperatures within multi-fin devices. Our numerical simulation data validates our parameter choices. We then introduce our thermal sensitivity metric, metric for electro-thermal sensitivity (METS), and investigate device sensitivities in different regions of operation. Our findings can be used to guide the design of optimal finFET devices, and to drive thermal-aware transistor and circuit-level optimizations.

The rest of this paper is organized as follows. We review device thermal analysis in Section II and propose our single-fin flared channel extension thermal model. In Section III, we model multi-fin devices and discuss critical design parameters in the thermal design of these devices. We then introduce our electro-thermal simulation methodology in Section IV. We provide experimental results in Section V and our METS metric in Section V-E. We describe some limitations of this work, and conclude with future research directions that highlight the role of thermal device modeling and its implication on circuit design.

\section{BACKGROUND: THERMAL ANALYSIS For SINGLE-FIN DEVICES}

Heat generated in n-type transistors is due to electron-phonon interactions in the drain region. When a device is turned on, free electrons in the source are accelerated through the channel to the

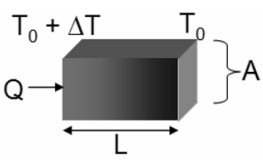

Fig. 3. Illustration of Fourier's law of heat conduction.

drain region. This acceleration causes the electrons to gain energy as they move through the channel. Once in the drain region, electrons are free to scatter with other electrons, phonons, impurity atoms, etc. Electron-phonon scattering results in an energy exchange between the electrons and the lattice which causes the lattice temperature to increase (other scattering mechanisms change electron momentum, but not energy) [19]. While electron-phonon scattering may occur in other regions of the device, any heat generated from the scattering produces an insignificant amount of heat generation when compared to the drain region.

Heat generated in the drain region of a finFET device causes a temperature gradient within the device. A detailed discussion of heat generation within transistors can be found in [19], [20]. An approximation of the generated heat (Watts) is

$$
Q=I_{D} \cdot V_{\mathrm{DS}} \text {. }
$$

The relationship between heat and temperature is governed by Fourier's law of heat conduction. This is illustrated in Fig. 3, where the boundary condition $T_{0}$ has been applied to the surface on right-hand side of the block. Fourier's law is

$$
\Delta T=\frac{L}{k \cdot A} \cdot Q
$$

where $\Delta T$ is temperature difference, $L$ is the length of the heat conduction, $k$ is the thermal conductivity of material in the heat conduction path, $A$ is the cross sectional area of heat conduction, and $Q$ is the heat. The electrical analogy of Fourier's law is Ohm's law. When heat is applied to a solid, a temperature gradient forms across the solid. This relationship is mathematically identical to an electrical current creating a voltage difference across and electrical resistor when forced through the resistor. If substitutions are made in (2) such that $\Delta T=\Delta V$, $Q=I$, and $L / k A=R$, the equation appears in the form of Ohm's law, $\Delta V=I \cdot R$. The equivalence between Fourier's law and Ohm's law is useful. Heat transfer analysis involving complicated geometries can be simplified by identifying select points within the geometry where temperatures are to be calculated. SPICE can then be used to solve for node voltages (i.e., temperatures) in the thermal network [21].

Pop et al. introduced a thermal model for an ultra-thin body SOI (UTB-SOI) device using the thermal-electrical equivalence [15]. The model uses a reduced thermal conductivity to account for the thin device geometry and impurity effects on the phonon mean free path. While not accounting for all thermal nano concerns, the model can be applied to devices with different gating structures, including finFETs. We refer to this model as the UTB model in the rest of the paper.

An ultra thin device and its equivalent UTB model are, respectively, shown in Figs. 4 and 5. The gate, drain, and source pads are assumed to connect through metal contacts to other circuit elements. Their top surface is assumed to be at a reference 


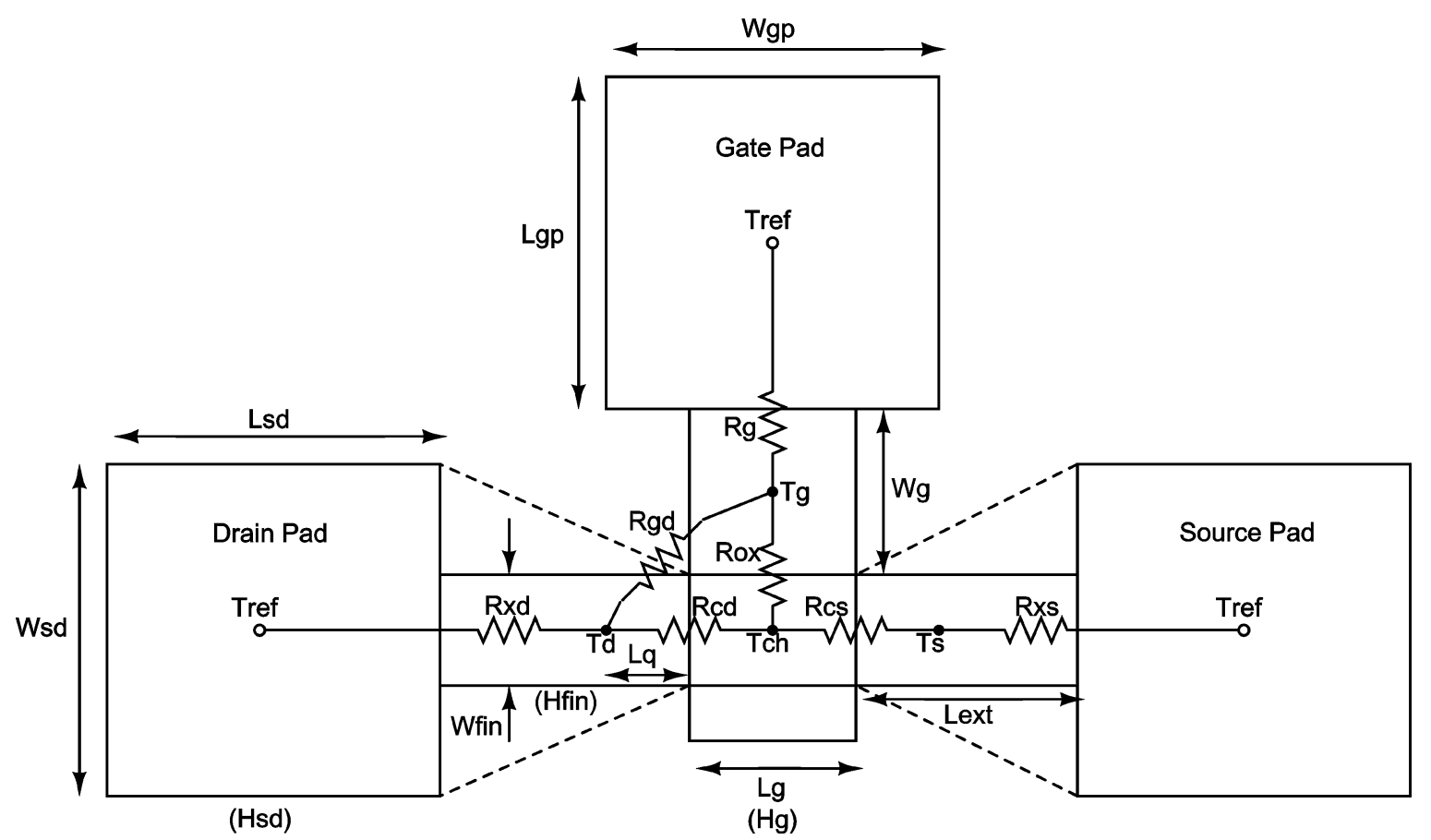

Fig. 4. Top view of a finFET layout with equivalent thermal resistances [15]. The dotted lines represent flared channel extensions. Markings with () signify height dimensions. Only one gate pad is used in this analysis.

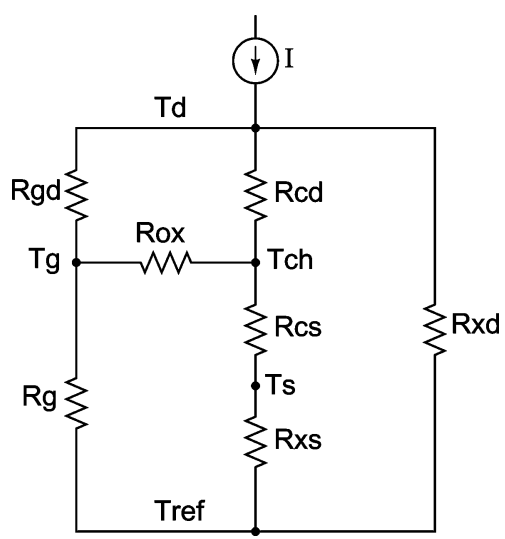

Fig. 5. Pop's equivalent thermal circuit [15].

temperature. ${ }^{1}$ Adiabatic boundary conditions are applied to all other surfaces. Thus, heat only flows in and out of the device at the top surface of the pads. Equivalent resistances are calculated using the formula $R=L / k A$, based on the materials and geometries through which heat transfer occurs. The current source representing the heat $Q$ can be applied to the UTB model at the drain node since it is the heat generation region. The injected current can be calculated using (1). Circuit analysis can then be used to solve for the temperatures at the drain, source, channel, and gate. Pop et al.'s findings showed that the device temperatures are most sensitive to the drain pad and channel extension dimensions.

${ }^{1}$ If the individual pad temperatures are known, the thermal model can be adjusted according by adding voltage sources between the source/drain/gate nodes and the reference temperature.

\section{A. Single-Fin Thermal Model Enhancement: Flared-Channel Extensions}

FinFET device performance is dependent on source and drain channel extension layout [22]. Flared channel extensions, as opposed to rectangular channel extensions, can be used to decrease parasitic source and drain channel extension resistance, hence improving $I_{\mathrm{on}}$. As fin thicknesses decreases, flared fins become more important in enhancing device performance. We enhance the UTB thermal model to account for flared channel extensions.

Fig. 4 shows two dashed lines on either side of the channel extension, representing the flared channel extension. We alter the source and drain channel extension thermal resistances $\left(R_{x d}\right.$ and $R_{x s}$ ) to properly model heat flow through the channel extension region. The channel extension region is sliced into $m$ segments from pad to channel and the thermal resistance of each segment is computed. The equivalent channel extension thermal resistance is the total series resistance the $m$ segments. Detailed flared-channel extension calculations will be shown in Section III.

\section{Multi-Fin Thermal Model}

To model wider finFETs with multiple fins, the equivalent thermal circuit model described in Section II is modified as follows. We assume that fins are spaced a distance $W_{\text {space }}$ apart, and that there will be two gate pads, one on each side of the outside-most fins. If an instance of Fig. 5 is used for each fin, only these outer fins can have the resistor $R_{g}$. An open circuit replaces $R_{g}$ for all inner fins. Furthermore, gate nodes of adjacent fins will be connected by an inter-gate thermal resistance, $R_{i}$, representing the heat flux path between fins through the polysilicon gate. This inter-gate resistance $R_{i}$ is calculated using $R=L / k A$, where $L$ is the fin separation $W_{\text {space }}, k$ is the 


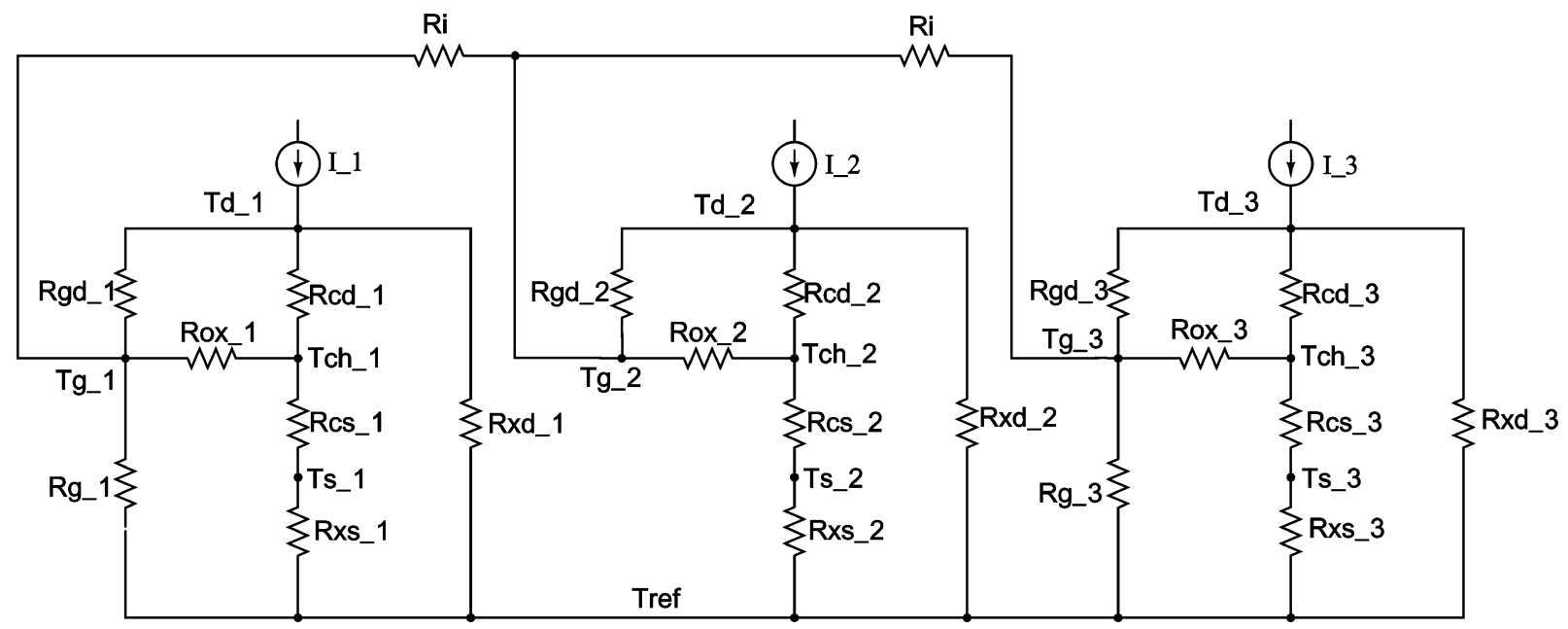

Fig. 6. Equivalent thermal circuit for a 3-fin finFET. All source, drain, and gate pads are at the reference temperature $\left(T_{\text {ref }}\right)$. All nodes and temperatures are indexed with $\_n$, where $n$ is the fin number (e.g., 1-3). The middle fin (index 2) gate resistance $\left(R_{g}\right)$ is replaced by the inter-gate thermal resistance $R_{i}$.

thermal conductivity of polysilicon, and $A$ is the cross sectional area of heat flow through the gate polysilicon. Fig. 6 shows an example thermal circuit of a 3-fin device. Heat injection occurs within the drain region for each fin.

To understand the effects of multi-fin device geometry on thermal characteristics, we analyze the equations used to generate the thermal resistance values. The thermal resistance from the source node to the source-side metal contact, $R_{x s}$, is dependent on the fin extension length and the size of the source pad. The resistance is computed as two series resistances. The first one is through the fin with the cross section of $W_{\text {fin }} \times H_{\text {fin }}$. The second resistance is between the fin and the top of the source pad. The heat flux in this region bends upwards from the area where the fin (cross section area of $W_{\text {fin }} \times H_{\text {fin }}$ ) meets the pad (cross section area of $L_{s d} \times H_{s d}$ ) to the metal on the top face of the pad. We assume that the length of the resistive path is about half of the length of the source pad, and that the cross section area of the path is the average of the two areas. The resistance $R_{x s}$ is then computed as

$R_{x s}=\frac{L_{\text {ext }}}{k_{\text {ext }} \cdot\left(W_{\text {fin }} \cdot H_{\text {fin }}\right)}+\frac{L_{s d}}{k_{s d} \cdot\left(W_{\text {fin }} \cdot H_{\text {fin }}+L_{s d} \cdot H_{s d}\right)}$.

The thermal resistance from the drain temperature node to the drain-side metal contact, $R_{x d}$, is dependent on the fin extension length as well as $L_{q}$. The latter parameter represents the centroid of heat generation region in the drain region [15]. It is the distance within the drain side of the fin from the edge of the gate. ${ }^{2}$ $R_{x d}$ is then computed as

$R_{x d}=\frac{L_{\mathrm{ext}}-L_{q}}{k_{\mathrm{ext}} \cdot\left(W_{\mathrm{fin}} \cdot H_{\mathrm{fin}}\right)}+\frac{L_{s d}}{k_{s d} \cdot\left(W_{\mathrm{fin}} \cdot H_{\mathrm{fin}}+L_{s d} \cdot H_{s d}\right)}$.

The thermal resistance from the center of the channel to the source thermal node, $R_{c s}$ is a function of half of the channel length

$$
R_{c s}=\frac{\frac{1}{2} L_{g}}{k_{c h} \cdot\left(W_{\text {fin }} \cdot H_{\text {fin }}\right)}
$$

${ }^{2} \mathrm{~A}$ heat generation region is not present in the source, therefore, $L_{q}$ only appears in drain side thermal resistance calculations.
$R_{c d}$, the thermal resistance from the center of the channel $\left(T_{c h}\right)$ to the drain thermal node $\left(T_{d}\right)$, is a series resistance of two resistances one involving half the channel length and the other involving the distance $L_{q}$

$$
R_{c d}=\frac{\frac{1}{2} L_{g}}{k_{c h} \cdot\left(W_{\text {fin }} \cdot H_{\text {fin }}\right)}+\frac{L_{q}}{k_{\text {ext }} \cdot\left(W_{\text {fin }} \cdot H_{\text {fin }}\right)} .
$$

Equation (7) shows the thermal resistance between the channel and the gate due to the gate oxide. It is dependent on the oxide thickness and also on $R_{\mathrm{if}}$, which is the interface resistance [15]. $R_{\text {if }}$ is independent of processing conditions and accounts for subtle boundary effects at the gate/oxide interface [15]. We assume that $t_{\mathrm{ox}}$ is the same as $t_{\mathrm{ox}-\mathrm{top}}$

$$
R_{\mathrm{ox}}=\frac{1}{L_{g}\left(2 \cdot H_{\mathrm{fin}}+W_{\mathrm{fin}}\right)}\left(R_{i f}+\frac{t_{\mathrm{ox}}}{k_{\mathrm{ox}}}\right)
$$

$R_{g}$, the thermal resistance between the gate poly at $T_{g}$ and the gate pad, is dependent on the distance of the gate pad from the fin $\left(W_{g}\right)$

$$
R_{g}=\frac{W_{g}}{k_{g} \cdot\left(L_{g} \cdot H_{g}\right)}+\frac{L_{g p}}{k_{g} \cdot\left(L_{g} \cdot H_{g}+L_{g p} \cdot H_{g}\right)} .
$$

Equation (9) shows the thermal resistance between the heat generation region $T_{d}$ and the gate temperature node. The thermal conduction path is through the oxide surrounding the device, which must be included due to the high temperature difference expected between drain and gate. A gate to source thermal resistance can be neglected due to the low temperature difference between the two regions and the small thermal conductivity of the oxide between the two regions

$$
R_{g d}=\frac{L_{q}}{\frac{1}{2} \cdot k_{\mathrm{ox}} \cdot W_{\mathrm{fin}} \cdot H_{g}} .
$$


Finally, the inter-fin gate resistance $R_{i}$ is calculated as follows, where $W_{\text {space }}$ is the spacing distance between two consecutive fins, $H_{g}$ is the gate height, and $L_{g}$ is the gate length: ${ }^{3}$

$$
R_{i}=\frac{W_{\text {space }}}{k_{g} \cdot H_{g} \cdot L_{g}}
$$

$R_{i}$, is likely to have a significant impact on the heat removal mechanism from the middle fins. It is directly proportional to $W_{\text {space }}$ and inversely proportional to $H_{g}$ and $L_{g}$. For the middle fins, two ways are possible for heat removal: through the source/ drain pads and through the gate.

The high thermal conductivity of the gate material provides the least resistive path for heat to flow out of the device. However, the small thermal conductivity of the oxide restricts heat flow from the channel to the gate. The small thermal conductivity of oxides (side, top, and buried) forces the heat to flow out of the source/drain extensions to the pads. Heat which does reach the gate material is free to spread throughout the device and exit through the gate pad.

The use of flared channel extensions alters the following resistances: $R_{x s}, R_{x d}$, and $R_{c d}$. The channel extension flare is assumed to start at the channel region and flare out the entire source/drain pad width as the dashed lines in Fig. 4 shows. We compute the flare resistances based on slicing the channel extension region into $m$ segments of varying dimensions [23]. The flared channel $R_{x s}, R_{x d}$, and $R_{c d}$ resistances are calculated as

$$
\begin{aligned}
R_{x_{-} \text {flare }}= & \frac{L_{s d}}{2 \cdot k_{s d} \cdot\left(L_{s d} \cdot H_{s d}\right)} \\
& +\frac{L_{\mathrm{ext}}}{m \cdot k_{\mathrm{ext}} \cdot H_{\mathrm{fin}}} \sum_{i=1}^{m} \frac{1}{W_{\mathrm{fin}}+\frac{2 \cdot i \cdot L_{\mathrm{ext}} \cdot \sin (\theta)}{m}} \\
R_{\text {xd_flare }}= & \frac{L_{s d}}{2 \cdot k_{s d} \cdot\left(L_{s d} \cdot H_{s d}\right)} \\
& +\frac{L_{\mathrm{ext}}}{m \cdot k_{\mathrm{ext}} \cdot H_{\text {fin }}} \sum_{i=n^{\prime}}^{m} \frac{1}{W_{\text {fin }}+\frac{2 \cdot i \cdot L_{\mathrm{ext}} \cdot \sin (\theta)}{m}} \\
R_{c_{-} \text {flare }}= & \frac{\frac{1}{2} L_{g}}{k_{c h} \cdot\left(W_{\mathrm{fin}} \cdot H_{\mathrm{fin}}\right)} \\
& +\frac{L_{\mathrm{ext}}}{m \cdot k_{\mathrm{ext}} \cdot H_{\mathrm{fin}}} \sum_{i=1}^{n^{\prime}} \frac{1}{W_{\mathrm{fin}}+\frac{2 \cdot i \cdot L_{\mathrm{ext}} \cdot \sin (\theta)}{m}} \\
\text { where: } \theta= & \tan ^{-1}\left(\frac{W_{s d}-W_{\mathrm{fin}}}{2 \cdot L_{\mathrm{ext}}}\right) \\
n^{\prime}= & \left\lfloor\frac{L_{q} \cdot m}{L_{\mathrm{ext}}}\right\rfloor \cdot
\end{aligned}
$$

By examining the previous equations, it is clear that the fin cross section, $H_{\text {fin }} \times W_{\text {fin }}$, affects most of the resistances. We conduct experiments in Section $\mathrm{V}$ to quantify the impact of variations of the following six parameters on electro-thermal behavior: $H_{\mathrm{fin}}, W_{\mathrm{fin}}, L_{g}, W_{\text {space }}, T_{\mathrm{ox}}$, and $H_{g}$.

\section{Electro-Thermal Device ANALysis}

Heat applied to the thermal circuit in Fig. 5 is dependent on the current flowing through the device as equation (1) describes. However, the current flowing through the device is dependent

\footnotetext{
${ }^{3}$ The gate-to-substrate thermal resistance is omitted from the model due to the small thermal conductivity of the underlying buried oxide.
}

on the device source temperature, due to the temperature dependence of mobility and threshold voltage. The source temperature controls carrier injection into the channel and ultimately limits the current flowing through the device [15], [24]. This leads to the natural mutual coupling of the electrical and thermal networks.

Electro-thermal simulation has been studied over the past several decades, with numerous approaches. The majority of these works target full chip electro-thermal simulations, requiring reduced thermal networks and/or simplified electrical models [25]-[30]. Unlike these target applications, we are interested in detailed device-level electro-thermal device analysis for finFETs. We have borrowed previous electro-thermal analysis techniques from Liu et al. [31] and Chiang et al. [32]. Liu et al. constructed electro-thermal device models which are thermally compensated for self heating by altering carrier mobility and threshold voltage using an RC equivalent thermal circuit. Chiang et al. used SPICE to solve a 3-D distributed thermal circuit model for interconnects. Their model accounts for interconnect self-heating and heat spreading to neighboring interconnects and layers.

Our electro-thermal methodology uses the electro-thermal simulation setup shown in Fig. 7 and updates all electrical temperature sensitive parameters, mainly mobility and threshold voltage, at every transient time step in SPICE. ${ }^{4}$ We couple the electrical and thermal circuits together through dependent sources, and then perform SPICE simulations. Our electro-thermal simulations allow us to simultaneously: 1) model the temperature effect within each fin on its current and 2) model the effect of current change on the temperature of each fin. We thus produce accurate drain, gate, source, and channel temperature estimations for each fin of a device while taking into consideration the exact location of the fin within a multi-fin device. In Section V, we show that this electro-thermal simulation is essential in avoiding the overestimation of the maximum fin temperatures and in accurately estimating the current in multi-fin devices.

\section{EXPERIMENTAL RESULTS}

Our goal is to evaluate the electro-thermal characteristics of multi-fin devices. Our baseline (nominal) device is a single fin with the parameters shown in Table I. ${ }^{5}$ Our data, when normalized, is in reference to this single-fin case. We first show the temperature and current profile of multi-fin device obtained using electro-thermal simulations. We then examine the temperature and current characteristics in rectangular and flared channel extensions. We then vary the fin geometries and investigate the impact of gate length and height, and fin width, height, and spacing on the temperature and performance of a multi-fin devices. Finally, we investigate the impact of temperature variations on device performance to obtain a thermal sensitivity metric. The experimental data presented herein uses adjusted model parameters with the electro-thermal simulation setup shown in Fig. 7, thus creating a new electrical and thermal device for each parameter under investigation.

${ }^{4}$ The finFET electrical models used in this work, BSIMDG [33], were obtained from Device Research Group at the University of California, Berkeley.

${ }^{5}$ The thermal conductivities $\left(k_{x x}\right)$ capture the reduced phonon mean free path due to thin device geometries and impurity effects based on Pop's findings [15]. 
TABLE I

MODEL FINFET DimEnsions AND THERMAL CONDUCTIVITIES

\begin{tabular}{||c|c|c|c|c|c|c|c|c|c||}
\hline$L_{g}$ & $H_{g}$ & $W_{g}$ & $H_{\text {fin }}$ & $W_{\text {fin }}$ & $t_{o x}$ & $L_{\text {ext }}$ & $L_{q}$ & $L_{s d}$ & $W_{\text {sd }}$ \\
\hline $50 \mathrm{~nm}$ & $75 \mathrm{~nm}$ & $140 \mathrm{~nm}$ & $65 \mathrm{~nm}$ & $10 \mathrm{~nm}$ & $16 \AA$ & $50 \mathrm{~nm}$ & $5 \mathrm{~nm}$ & $200 \mathrm{~nm}$ & $200 \mathrm{~nm}$ \\
\hline \hline$H_{s d}$ & $L_{g p}$ & $W_{g p}$ & $W_{\text {space }}$ & $R_{i f}$ & $k_{g}$ & $k_{c h}$ & $k_{\text {ext }}$ & $k_{o x}$ & $k_{\text {sd }}$ \\
\hline $65 \mathrm{~nm}$ & $200 \mathrm{~nm}$ & $200 \mathrm{~nm}$ & $100 \mathrm{~nm}$ & $20 e^{-9} \frac{\mathrm{m}^{2} \cdot \mathrm{K}}{\mathrm{W}}$ & $45.3 \frac{\mathrm{W}}{\mathrm{m} \cdot \mathrm{K}}$ & $6.5 \frac{\mathrm{W}}{\mathrm{m} \cdot \mathrm{K}}$ & $13.0 \frac{\mathrm{W}}{\mathrm{m} \cdot \mathrm{K}}$ & $1.38 \frac{\mathrm{W}}{\mathrm{m} \cdot \mathrm{K}}$ & $13.0 \frac{\mathrm{W}}{\mathrm{m} \cdot \mathrm{K}}$ \\
\hline
\end{tabular}

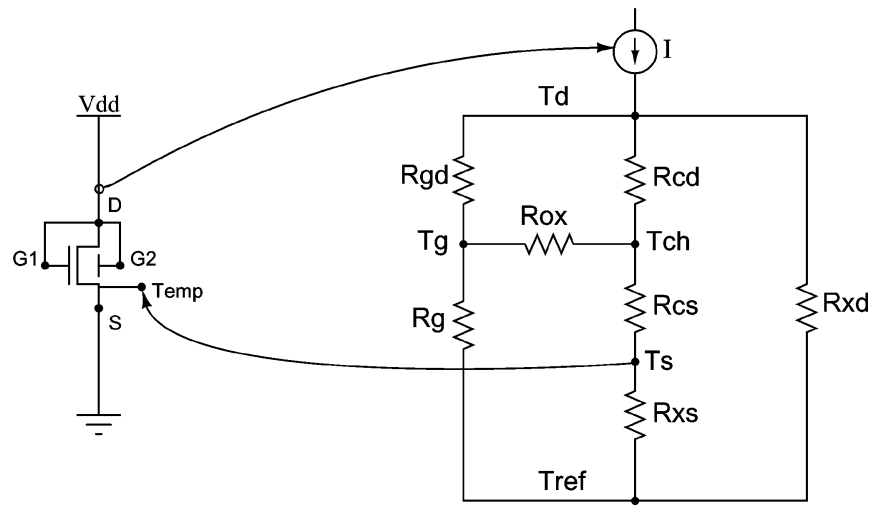

Fig. 7. Equivalent electro-thermal finFET model.

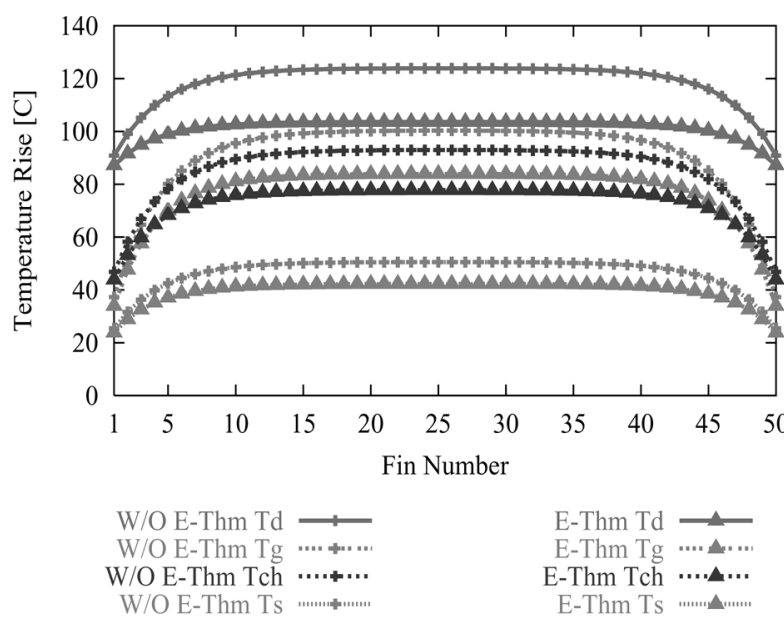

Fig. 8. Temperature profile at the source $\left(T_{s}\right)$, channel $\left(T_{c h}\right)$, gate $\left(T_{g}\right)$, and drain $\left(T_{d}\right)$, for a 50-fin device. We show the temperatures obtained with (E-Thm) and without (W/O E-Thm) electro-thermal simulations.

\section{A. Multi-Fin Electro-Thermal Analysis}

Using the multi-fin thermal model with electro-thermal simulations, we examine: fin temperatures and currents. We also compare the temperature of multi-fin devices with rectangular and flared channel extensions.

1) Multi-Fin Temperature Profiles in Rectangular Channel Extension Devices: We construct multi-fin devices assuming a fin spacing, $W_{\text {space}}$, of $100 \mathrm{~nm}$. A plot of temperature rise (above ambient) for each fin of a 50-fin device is shown in Fig. 8. Several observations can be made. First, the inner fins are hotter than outer ones, for the drain, source, gate, and channel. Each fin has the same access to the source/drain pads; however, the gate pads at the reference temperature are further away from the inner fins. This is also the cause for the gate temperature for the inner fins to be hotter than the channel temperature. The gate

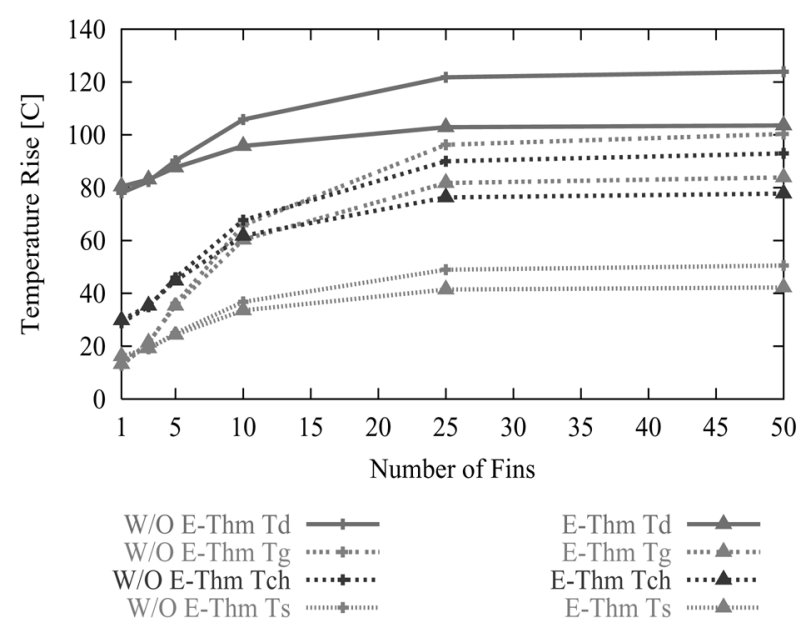

Fig. 9. Temperature dependence (at the source $\left(T_{s}\right)$, channel $\left(T_{c h}\right)$, gate $\left(T_{g}\right)$, and drain $\left(T_{d}\right)$ ) on the number of fins, demonstrated for 1-, 3-, 5-, 10-, 25-, and 50-fin devices. We show the temperatures obtained with (E-Thm) and without (W/O E-Thm) electro-thermal simulations.

pads are effective at removing the heat from the gates for outer fins, but less so for the inner fins. Second, for the majority of inner fins, the temperature is relatively constant from one fin to the next. Thus, beyond a certain number of fins, adding more fins to a device will no longer increase the peak temperatures. Third, the drain temperature is the hottest, and the coolest is the source. However, the drain temperature, while the hottest part of the finFET, has the smallest variation across the fins.

The peak temperatures of devices with $1,3,5,10,25$, and 50 fins are compared in Fig. 9. The results are consistent results with those drawn from Fig. 8. Indeed, the maximum temperature at the drain, source, channel, and gate increases with a larger number of fins, but reaches steady state at or beyond 25 fins. For the single-fin device, the source temperature is at a higher temperature than the gate. However, when the device has three or more fins, the gate temperature exceeds that of the source. The peak temperature at the drain exhibits the smallest variations across all the examined devices.

2) $I_{\text {on }}$ in Multi-Fin Devices: We performed an experiment to validate the need to model the co-dependence of current and heat injection in finFETs. Fig. 9 contrasts peak temperatures obtained using electro-thermal simulations against those obtained assuming uniform temperatures for all the fins. In the latter case, we assume the heat injection at each fin is equal to that of a single fin obtained using electro-thermal analysis. In both cases, a $25^{\circ} \mathrm{C}$ ambient temperature was assumed. Ignoring the dependence of the current and thus the heat injection on temperature leads to the gross overestimation of the multi-fin temperature profiles. This is true for all four regions, and worst for the drain $\left(\sim 20^{\circ} \mathrm{C}\right)$. 


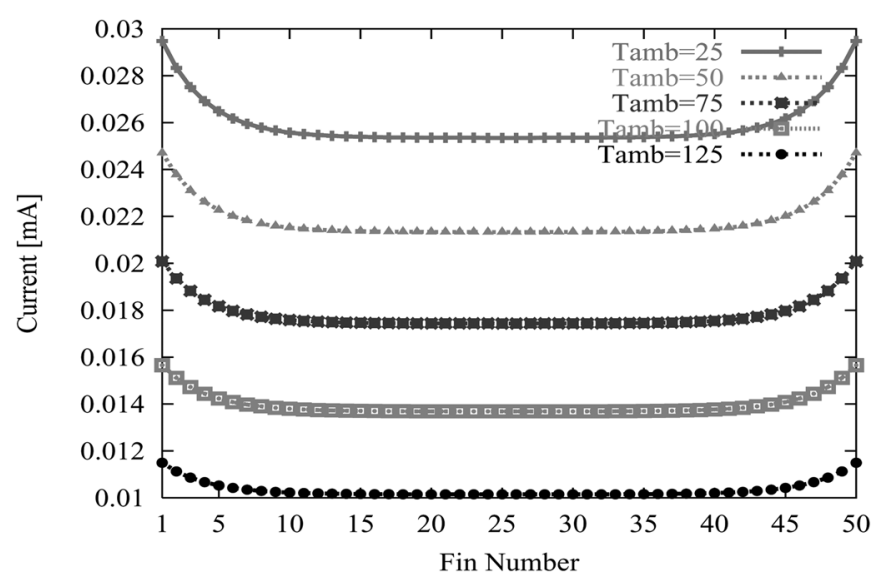

Fig. 10. Current versus fin number for 50-fin device at different ambient temperatures.

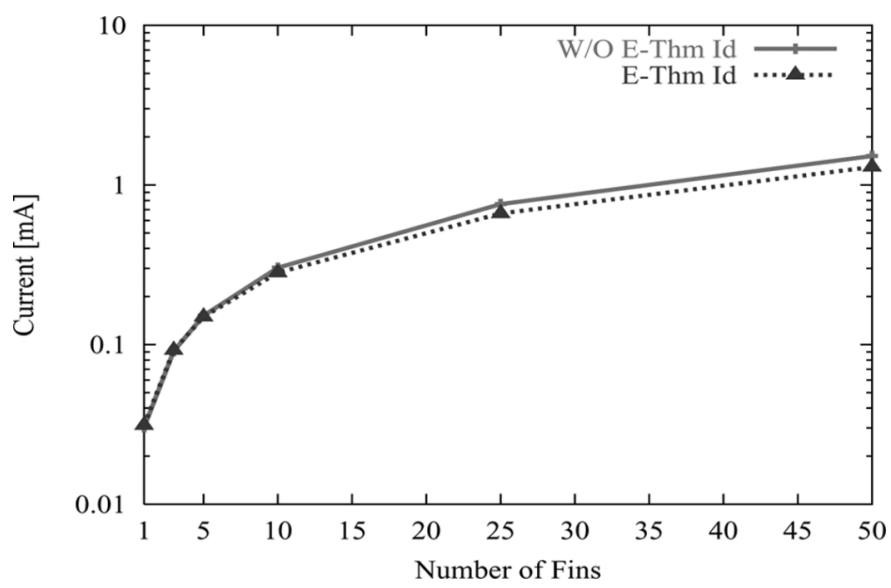

Fig. 11. Current for 1-, 3-, 5-, 10-, 25-, and 50-fin devices. E-Thm uses electrothermal simulations, while W/O E-Thm assumes uniform temperatures (that of a single fin obtained via electro-thermal simulation) across all fins.

Fig. 8 shows a temperature profile for a 50-fin device. The temperature difference across the fins directly impacts the current through each fin. Using electro-thermal simulations, we examine the current through each fin of a 50-fin device in Fig. 10 at different ambient temperatures. As expected, the outer fins carry more current than the inner fins. Equally interesting, the $\Delta I$ for the outer versus inner fins decreases as the ambient temperature increases. This suggests the impact of fin-to-fin interactions diminish at high ambient temperatures.

Fig. 11 shows the current of multi-fin devices with and without electro-thermal coupling. Electro-thermal coupling uses the individual fin temperature to compute the current through each individual device fin. Currents obtained without electro-thermal coupling assume a uniform temperature (that of a single-fin device) across all fins. The result is lower device temperatures, smaller gradients across the device, and reduced current. The overestimation is less prevalent with fewer fins as the temperature differences across the regions are less acute. The overestimation approaches a constant value for devices with more than 25 fins. From our plot, assuming constant heat injection over predicts current flow through the device by $\sim 15 \%$ and $\sim 7 \%$ for 50 -fin and 10-fin devices, respectively.

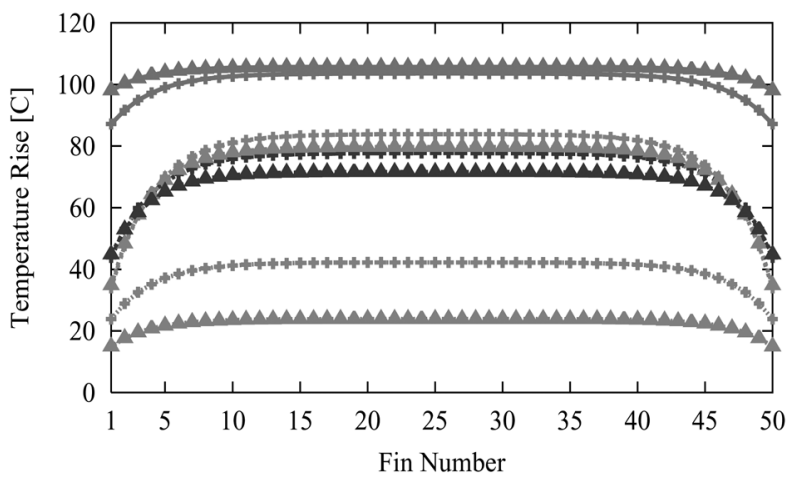

$$
\begin{aligned}
& \text { Td - Rect. Fin } \longrightarrow \\
& \text { Tg - Rect. Fin } \cdots+\cdots+\cdots \\
& \text { Tch - Rect. Fin } \cdots \cdot+\cdot \cdot \\
& \text { Ts - Rect. Fin …ㄴ.."… } \\
& \text { Td - Flare Fin } \longrightarrow \\
& \text { Tg - Flare Fin .... } \\
& \text { Tch - Flare Fin ........ } \\
& \text { Ts - Flare Fin }
\end{aligned}
$$

Fig. 12. Temperature profile at the source $\left(T_{s}\right)$, channel $\left(T_{c h}\right)$, gate $\left(T_{g}\right)$, and drain $\left(T_{d}\right)$, for a 50-fin device. The rectangular channel extension device is represented by Rect. Fin, while the flared channel extension device is shown as Flare Fin.

\section{B. Flared vs. Rectangular Channel Extension Comparison}

Flared channel extensions increase $I_{\text {on }}$ by decreasing parasitic source/drain resistance. The current increase results in self-heating within the device. This section explores the thermal differences between rectangular and flared channel extensions.

Fig. 12 shows two 50-fin device temperature profiles: one with rectangular channel extensions and one with flared channel extensions. The flared channel extension multi-fin thermal model discretized the flared source and drain regions into 100 segments. ${ }^{6}$ Several observations can be made when studying Fig. 12. First, the maximum device temperature $\left(T_{d}\right)$ is higher in the flared channel extension device due to a larger $I_{\mathrm{on}}$. Second, the fin-to-fin $\Delta T$ is less pronounced in the flared channel extension device. Third, the maximum channel and gate temperatures are relatively close to one another, but deviate significantly at the outer fins. Finally, the source temperature for the flared channel extension is less than the rectangular channel extension source temperature. This can be attributed to the lower source/drain channel extension thermal resistances. The reduced thermal resistances allow more heat to flow out of the drain extension region to the drain pad than into the device. Heat flow which does reach the source regions also has a less restrictive heat flow path to the source pad, thus reducing the source temperature. Flared channel extensions not only reduce source/drain electrical resistance, but also reduce the source-to-pad thermal resistance resulting in a cooler source temperature, thus further increasing $I_{\mathrm{on}}$.

Fig. 13 compares maximum device temperatures for the source, channel, gate, and drain regions of rectangular and flared channel extension devices. Complementing the observations made in Figs. 9 and 12, one additional conclusion can be drawn from the figure. The drain and source temperatures differences across all device sizes in the flared channel extension devices are less pronounced than in the rectangular channel extension devices, due to the lower thermal resistance of the channel extensions. Less heat flows through the gate

\footnotetext{
${ }^{6}$ The electrical model also accounts for the reduced source/drain channel extension resistance by altering the parasitic source/drain resistance.
} 


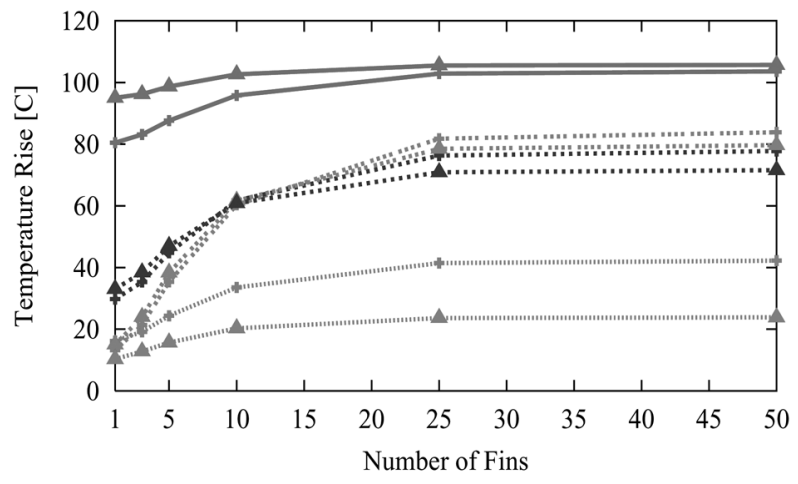

$$
\begin{aligned}
& \text { Td - Rect. Fin } \\
& \text { Tg - Rect. Fin ....+*... } \\
& \text { Tch - Rect. Fin …+... } \\
& \text { Ts - Rect. Fin num..... } \\
& \text { Td - Flare Fin } \\
& \text { Tg - Flare Fin ..... ㅅ..... } \\
& \text { Tch - Flare Fin ......... } \\
& \text { Ts - Flare Fin }
\end{aligned}
$$

Fig. 13. Temperature dependence (at the source $\left(T_{s}\right)$, channel $\left(T_{c h}\right)$, gate $\left(T_{g}\right)$, and drain $\left.\left(T_{d}\right)\right)$ on the number of fins, demonstrated for 1-, 3-, 5-, 10-, 25-, and 50-fin devices. The different channel extension configuration are represented as Rect. Fin and Flare Fin.

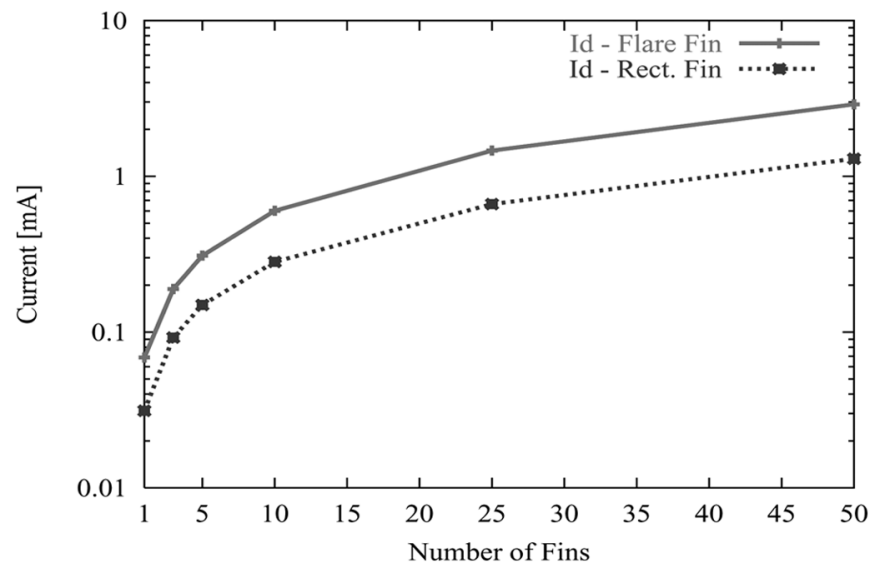

Fig. 14. Current dependence on the number of fins, demonstrated for 1-, 3-, 5-, 10-, 25-, and 50-fin devices. The different channel extension configurations are represented as Rect. Fin and Flare Fin.

polysilicon to the gate pad, reducing the effect of moving the gate pad further away from the inner fins, as shown in Fig. 12.

Fig. 14 shows the current dependence on the number of fins for rectangular channel extensions and flared channel extensions. Flared channel extensions reduce source/drain parasitic resistance and increase $I_{\mathrm{on}}$, as previously mentioned. Flared channel extensions increase $I_{\text {on }}$ over rectangular channel extension devices by $\sim 1.9 x$ to $\sim 2.0 x$ for single-fin and 50 -fin devices.

\section{Impact of Device Geometries}

In our next set of experiments, we quantify the effects of the following parameters: $L_{g}, W_{\text {space }}, H_{g}, T_{\text {ox }}, W_{\text {fin }}$, and $H_{\text {fin }}$ on finFET temperature and performance. We vary each parameter as follows. For $L_{g}$, we examine several gate lengths between 25 and $100 \mathrm{~nm}$. For fin spacing, $W_{\text {space }}$, we chose 50, 100, 200, and $400 \mathrm{~nm}$. For the rest of the parameters we varied them by $\pm 10 \%$, and $\pm 20 \%$ as that would reflect some processing variations.

1) Gate Length Variations: Fig. 21(a) plots the maximum drain temperature for multi-fin devices that have 1, 3, 5, 10, 25, and 50 fins, while varying the device length, $L_{g}$ for rectangular

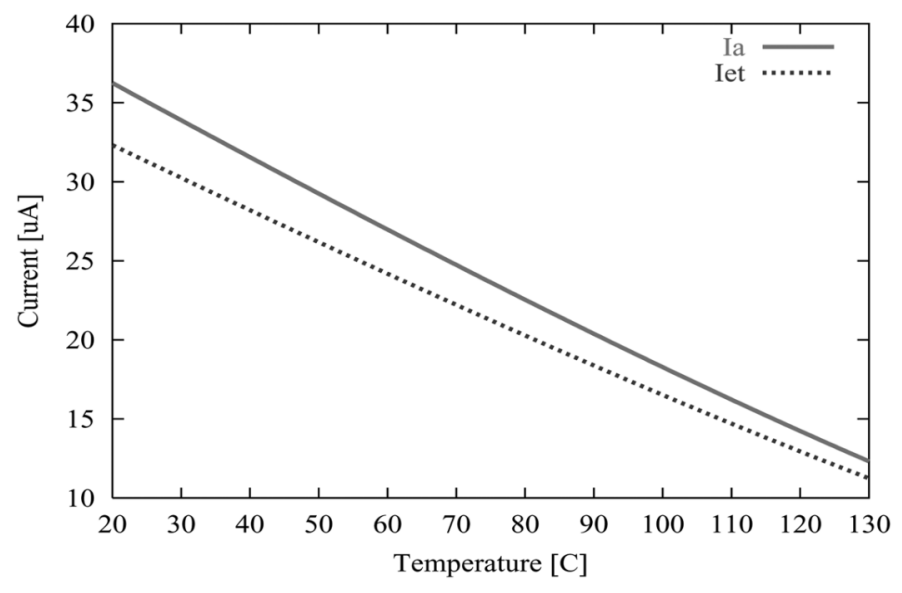

Fig. 15. Current dependence on temperature for single-fin device with rectangular channel extensions. The $x$-axis temperature represents the ambient temperature.

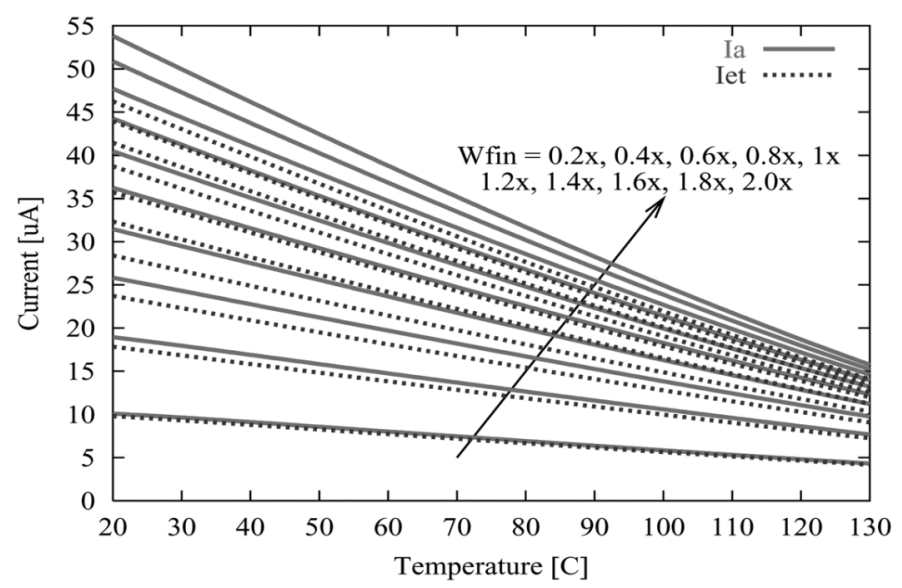

Fig. 16. Current dependence on temperature for single-fin device with rectangular channel extensions for nominal fin thickness variations of $0.2 x$ to $2 x$.

channel extensions and flared channel extensions respectively. Fig. 21(a) shows a single-fin temperature difference of $\sim 5 \%$ between the smallest and largest gate lengths for rectangular channel extensions and a $12 \%$ difference for flared channel extensions.

The small temperature variations in the drain are due to large temperature fluctuations in the source as demonstrated in Fig. 19(a) for a rectangular channel extension device. Longer devices typically produce less drive current than shorter devices. However, the thermal resistance between the drain and source is larger in longer devices than in shorter devices. The effect of increased in channel thermal resistance can be inferred from Fig. 21(a). As the gate length in rectangular channel extensions increases the channel resistance significantly impedes heat flow from the drain to source/gate pads and thus forces all heat to flow out of the drain extension into the drain pad. However, the impact of increased gate length on flared channel extensions is less severe, as the majority of heat flows out of the drain extension into the drain pad as opposed to flowing through the channel to the source/gate pads. The decreased heat flow from drain to source also causes the source to operate at a lower temperature. The lower temperature increases the current in longer devices, whereas higher temperatures degrade the 


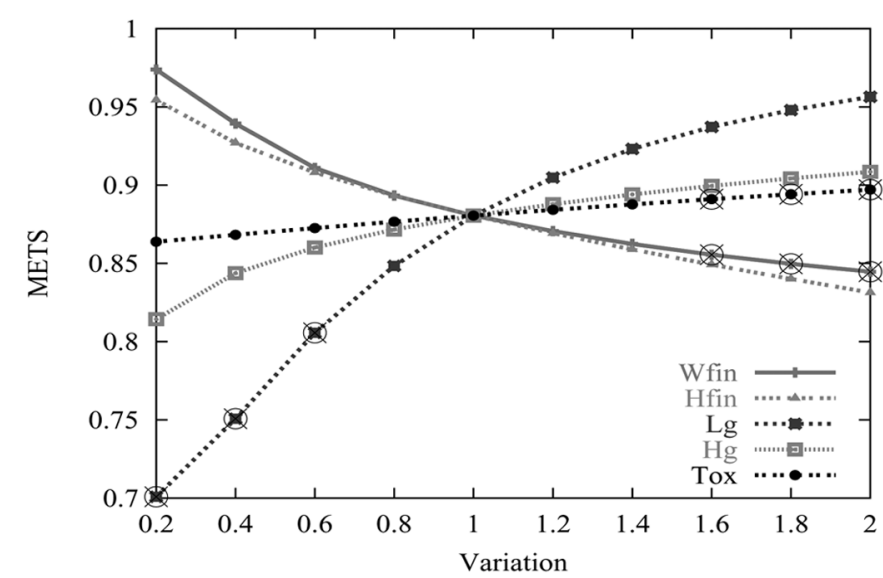

(a)

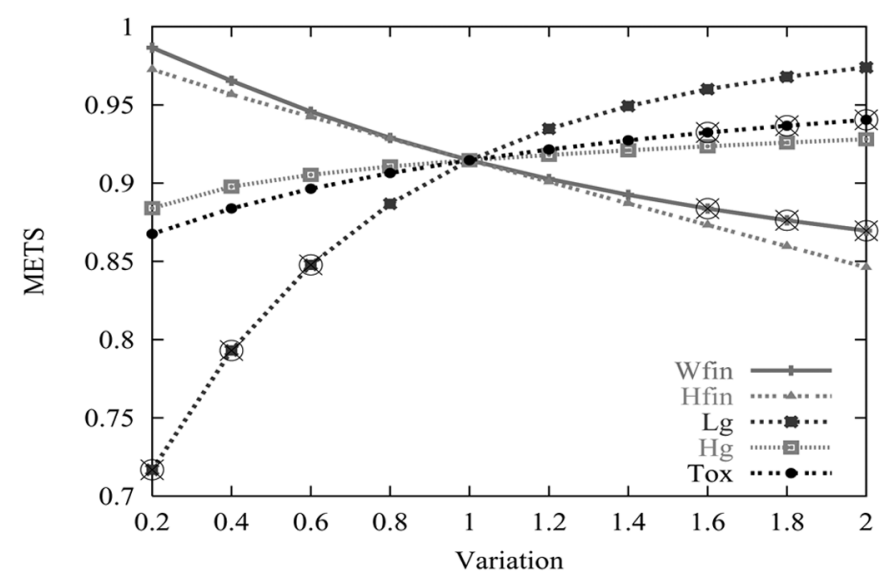

(b)

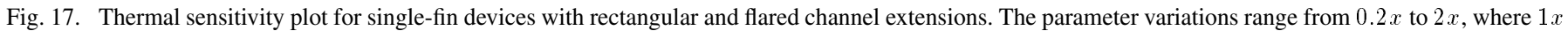

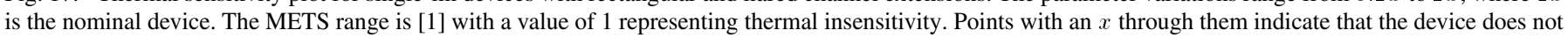
conform to the fin electrical design recommendations. (a) Rectangular channel extension. (b) Flared channel extension.

current in shorter devices. This electro-thermal phenomenon is demonstrated in Fig. 19(b) for channel lengths of 100, 50, and $25 \mathrm{~nm}$. The difference in current between the 25 and 100 $\mathrm{nm}$ devices is $\sim 17 \%$ and $\sim 11 \%$ for 50 -fin and 10 -fin devices respectively. Thus, reduced gate lengths do not substantially increase drive current due to the thermal overheads associated with shorter gate lengths.

2) Fin Spacing Variations: We next examine the effect of varying the fin spacing, $W_{\text {space, }}$ on the drain temperature. The results are reported in Fig. 21(b) for rectangular and flared channel extensions. One would expect that more tightly packed fins to have higher temperatures; however, this is only true for large flared channel extension devices. The tighter spacing reduces $R_{i}$ between the fins and effectively reduces the gate to pad resistance for each fin. This leads to smaller maximum temperatures as the heat removal path through the gate is less resistive than it is with wider spacing. This indicates that the maximum temperature in each drain is mostly due to electron-phonon interactions rather than heat spreading from neighboring fins. For larger fin spacing, the maximum drain temperature is achieved via few fins: the maximum temperature is almost achieved with a 10-fin device. The important conclusion here is that packing the fins tightly could potentially help with the heat removal. However, the approximation of the heat removal capabilities of the environment (i.e., ignored in this study) is needed to verify this conclusion.

3) Gate Height Variations: In our next experiment, the effect of varying gate height $\left(H_{g}\right)$ on maximum device temperature is quantified. The maximum device temperature versus number of fins for gate height changes of $\pm 20 \%$ nominal is shown in Fig. 21(c) for rectangular and flared channel extensions. The effect of varying $H_{g}$ is more evident for devices with a smaller number of fins. Varying $H_{g}$ has less than a $10 \%$ change on the temperatures. Again, this indicates that the heat removal is more prevalent through the source/drain pads than through the gate. This is in support of Pop et al.'s findings that the dimensions of the source and drain are of critical importance [15].

4) Oxide Thickness Variations: We next investigate the impact of oxide thickness variations, $T_{\mathrm{ox}}$, on device tempera- ture and current. The thermal results are shown in Fig. 21(d) while the electrical results are shown in Fig. 20(a). Varying $T_{\text {ox }}$ by $\pm 20 \%$ produces a $\sim 7 \%$ change in temperature and a $\sim 6 \%$ change in current for the single-fin rectangular channel extension device. Results for the flared channel extension device are more dramatic. Changing $T_{\text {ox }}$ by $\pm 20 \%$ results in a $\sim 20 \%$ change in temperature and a $\sim 15 \%$ change in current. The changes in temperature remain relatively constant across different device types and sizes, as shown in Fig. 21(d). From a device standpoint, $T_{\text {ox }}$ variations have a greater impact on device electrostatics than the device thermal properties. The reason for this is that 1) $T_{\mathrm{ox}}$ controls the $V_{T}$ of the device and ultimately effects the current through the device and 2) the thermal conductivity of the oxide $\left(k_{\mathrm{ox}}\right)$ is much smaller than the other device thermal conductivities $\left(k_{g}, k_{\mathrm{ch}}, k_{\mathrm{ext}}\right.$, etc.).

5) Fin Width Variations: Our next experiment examines how $W_{\text {fin }}$ affects drain maximum temperature, shown in Fig. 21(e), for rectangular and flared channel extensions. Changes in fin width affect almost all thermal resistances in the circuit, resulting in significant changes in maximum device temperature for fin width changes of $\pm 20 \%$. Increasing the fin width reduces thermal and electrical resistance between the source/drain to the pads, which reduces device temperature and increases $I_{\text {on }}$. Fig. 21(e) shows a $~ 7 \%$ difference in maximum temperature for rectangular channel extensions and a $\sim 17 \%$ difference in temperature for flared channel extensions.

6) Fin Height Variations: Our final study examines the impact of $H_{\text {fin }}$ on drain temperature and device current as shown in Fig. 21(f) and in Fig. 20(b). Increasing $H_{\text {fin }}$ ultimately increases the device current as the device width (per fin basis) is defined by $H_{\text {fin }}$. The increase in current results in greater heat generation and higher device temperatures as Fig. 21(f) shows. Varying $H_{\text {fin }}$ by $\pm 20 \%$ yields a $\sim 13 \%$ change in temperature and a $\sim 45 \%$ change in current for a single-fin device with rectangular channel extensions and a $20 \%$ change in temperature with a $\sim 44 \%$ change in current for the same device with flared channel extensions. Changing the fin height produces the most variation in temperature among all the other parameters examined. This is because the fin height affects almost all of the thermal resis- 


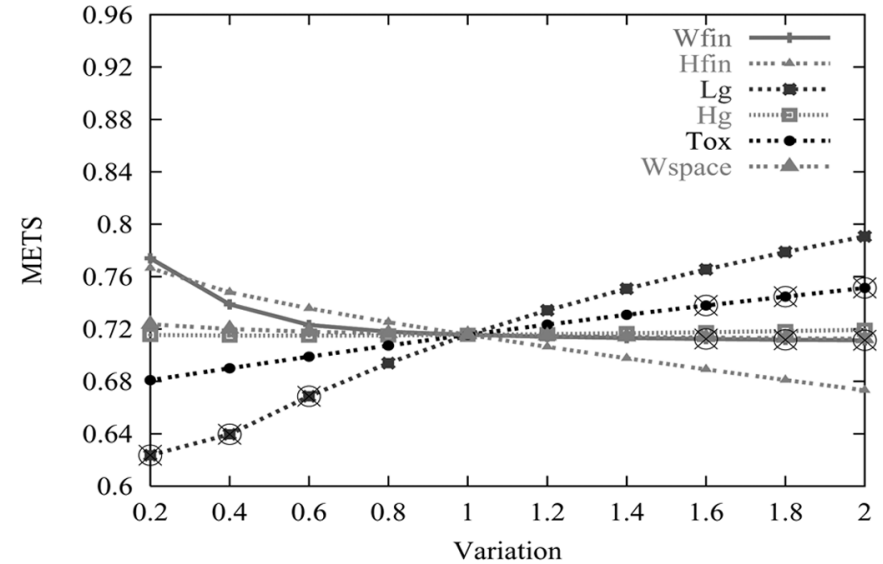

(a)

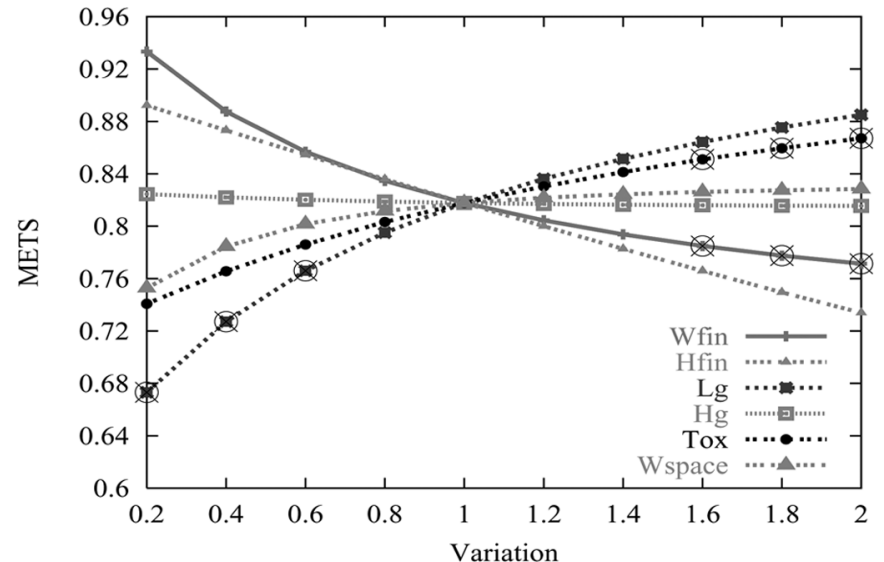

(b)

Fig. 18. Thermal sensitivity plot for 50 -fin devices with rectangular and flared channel extensions. The parameter variation ranges from $0.2 x$ to $2 x$ a nominal device. The METS range is [1] with a value of 1 representing thermal insensitivity. Points with an $x$ through them indicate that the device does not conform to the fin electrical design recommendations. (a) Rectangular channel extension device. (b) Flared channel extension device.

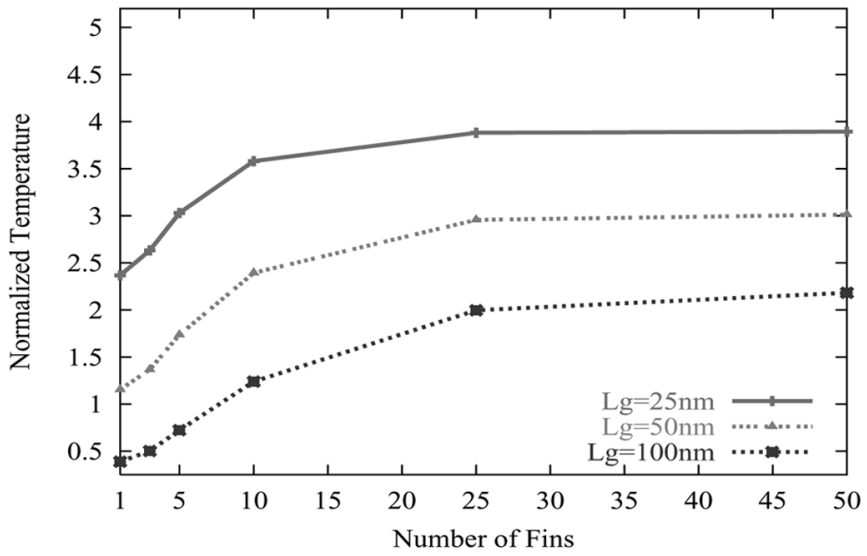

(a)

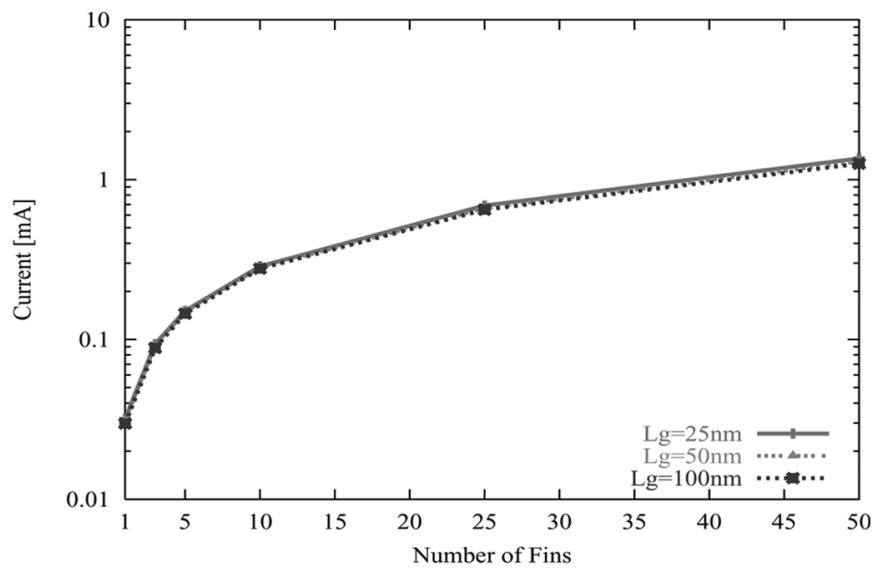

(b)

Fig. 19. Source temperature and current dependence on gate length for rectangular channel extension devices. (a) Source temperature dependence on Lg. (b) Current dependence on Lg.

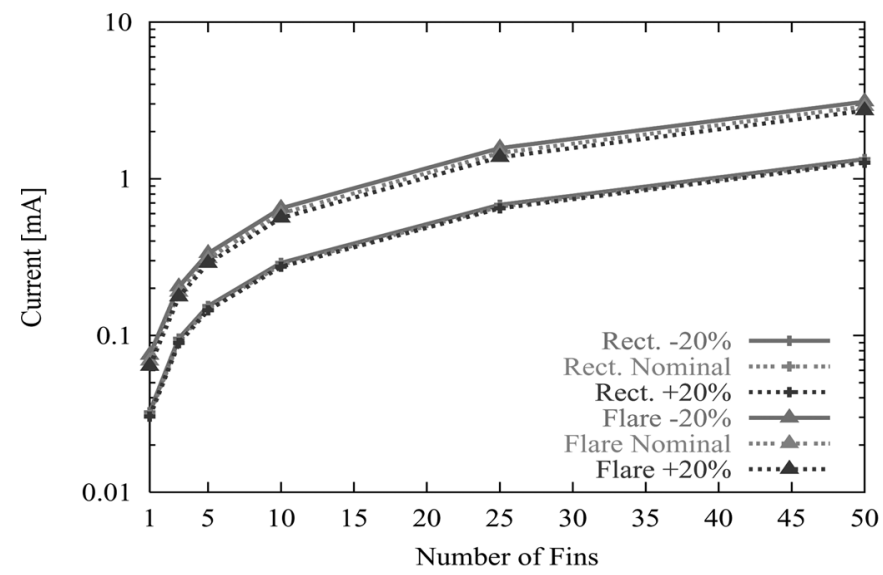

(a)

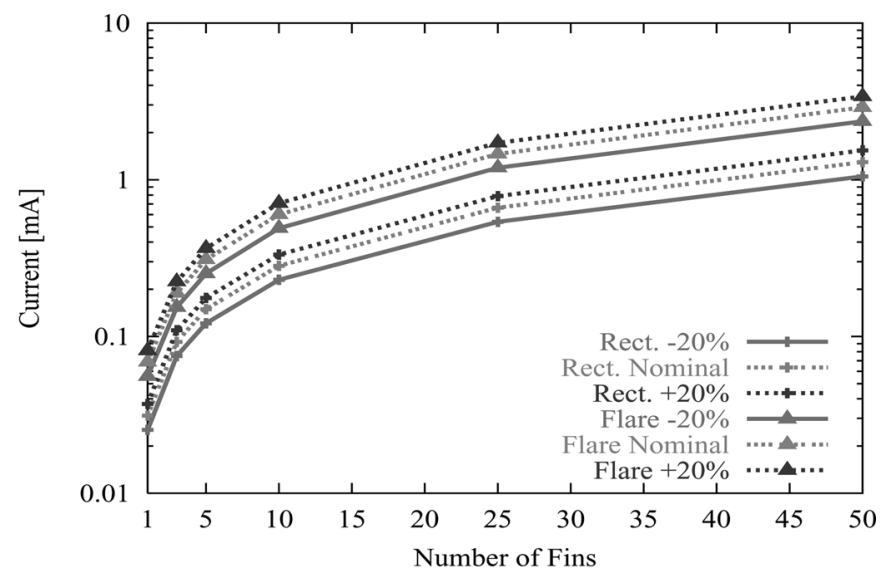

(b)

Fig. 20. Current for 1-, 3-, 5-, 10-, 25-, and 50-fin devices for different oxide thicknesses and fin heights in rectangular channel extension devices (Rect.) and flared channel extension devices. (a) Current dependence on $T_{\text {ox }}$. (b) Current dependence on $H_{\text {fin }}$. 
tance values associated with the circuit. A taller fin reduces $R_{x s}$, $R_{x d}, R_{c s}, R_{c d}$, and $R_{\mathrm{ox}}$. This in turn reduces the temperature differential between the drain and source regions. The resulting source temperature is reduced, resulting in increased drive current $\left(I_{\text {on }}\right)$.

\section{Thermal Sensitivity of FinFETs}

In Section V-C, we examined how changes in device parameters affect device temperature and current. All of our comparisons were carried out at a single ambient temperature. In this section, we examine how temperature in conjunction with variations in device parameters alter device performance.

We begin by looking at a current versus temperature for a nominal single-fin device. Fig. 15 illustrates the current in the nominal finFET device with and without electro-thermal simulations. $I_{a}$ is the current obtained assuming the fin is at the ambient temperature (the $x$-axis in Fig. 15). $I_{e t}$ is the current obtained using electro-thermal simulations assuming the ambient temperature is the $x$-axis temperature in Fig. $15 . I_{e t}$ reflects device self-heating as well as the ambient temperature. The figure illustrates the overestimation of the current without using the electro-thermal simulation. At $20^{\circ} \mathrm{C}$, the difference between the two simulations is $\sim 11 \%$. As the ambient temperature rises it becomes dominant over self-heating (the $\Delta I$ between $I_{a}$ and $I_{e t}$ decreases). Thus, the amount of self-heating depends on the ambient temperature. Fig. 15 also allows the total self-heating to be calculated. At a given current, the difference in temperature between $I_{a}$ and $I_{e t}$ is due to self-heating. For example, a current of $30 \mu \mathrm{A}$ produces $\sim 17^{\circ} \mathrm{C}$ of self-heating. ${ }^{7}$

Geometric parameter variations influence device current and self-heating. Given a set of geometric parameter variations, either process or design, the thermal sensitivity can be calculated to determine robustness to self-heating. Fig. 16 shows $I_{a}$ and $I_{e t}$ for fin thickness variations of $0.2 x$ nominal to $2 x$ nominal $W_{\text {fin }}$. As Fig. 16 shows, the thermal sensitivity is strongly dependent on the fin thickness variation. We focus on the $0.2 x$ nominal fin thickness. Two observations can be made. First, the currents, $I_{a}$ and $I_{e t}$, as well as the difference between the two currents are reduced when compared to the nominal device in Fig. 15. Second, the $\Delta I$ across all ambient temperatures remains constant, producing parallel $I_{a}$ and $I_{e t}$ versus temperature lines. The parallel lines indicate constant self-heating across all ambient temperatures, resulting in a thermally robust device.

\section{E. METS}

Characterizing thermal sensitivity by $I_{a}$ and $I_{e t}$ becomes infeasible for multiple parameters with large variations due to the enormous amount of data required to evaluate the devices, as demonstrated in Fig. 16. Thus, a metric which summarizes the electro-thermal data is required. The metric must have the following characteristics.

- For the metric to be useful, it should not depend on a particular operating temperature.

- The metric should be able to capture the effects of device processing and geometries.

\footnotetext{
${ }^{7}$ All temperatures are in reference to the source temperature, as the source temperature controls carrier injection into the channel and ultimately $I_{\text {on }}[15]$, [24].
}

- The metric must be independent of the method used to obtain the underlying simulation results as we wish the metric to remain valid with advances in simulation and modeling technologies.

- It is desirable that the metric is general and can be applied to a wide range of devices, thus allowing useful comparisons about device sensitivities in different regions of operation.

Our metric, METS, measures device thermal robustness by summarizing the $I_{a}$ and $I_{e t}$ simulation data [34]. To establish the metric, we utilize the difference between $I_{a}$ and $I_{e t}$ at different temperatures. Each simulation $\left(I_{a}\right.$ and $I_{e t}$ pair) in Fig. 16 is characterized by a sensitivity slope, $S_{a}$ or $S_{e t}$, over a wide range of operation. The sensitivity slope reflects the change in current due to temperature changes. In the case of $S_{a}$, the slope captures changes in ambient temperature. For $S_{e t}$, the slope captures temperature due to both self-heating and ambient temperature. The self-heating metric can be expressed as $S_{e t} / S_{a}$ for each device. METS is the computed as

where

$$
M E T S=\frac{S_{e t}}{S_{a}}
$$

$$
\begin{aligned}
S_{e t} & =\frac{I_{e t_{T_{2}}}-I_{e t_{T_{1}}}}{T_{2}-T_{1}} \\
S_{a} & =\frac{I_{a_{T_{2}}}-I_{a_{T_{1}}}}{T_{2}-T_{1}} .
\end{aligned}
$$

METS is confined to the range of [1]. A device with constant self-heating will have METS equal to one, that is, $I_{a}$ and $I_{e t}$ will be parallel. However, a device with substantial self-heating will have a METS ratio less than one. The temperatures, $T_{1}$ and $T_{2}$, are selected based on the expected operating range of the device and the $I_{a} / I_{e t}$ relationship. Selecting $T_{1}$ and $T_{2}$ at the extreme temperatures of the operating region is sufficient for a linear $I_{a} / I_{e t}$ relationship. When the $I_{a} / I_{e t}$ relationship is nonlinear, the operating range can be decomposed into multiple temperature regions and the METS can be found for each of the regions. The nominal device shown in Fig. 15 has a METS ratio of 0.88. Fig. 17(a) shows the METS ratio for a single-fin rectangular channel extension device with several geometric $\left(W_{\text {fin }}\right.$, $H_{\text {fin }}, L_{g}, H_{g}$, and $T_{\text {ox }}$ ) variations. The baseline (nominal) device is the single-fin rectangular channel extension device with the parameters shown in Table I. Each point on the graph represents the METS for a given deviation from a nominal device, referred to as $1 x$. Fig. 17(b) illustrates the METS ratio of a flared channel extension device. For a nominal device, the METS ratio of a flared channel extension device is higher than that of a rectangular channel extension device ( 0.91 versus 0.88$)$. This indicates that the flared channel extension is effective in reducing the thermal sensitivity of finFETs. The flared channel extension decreases both the thermal and electrical resistance of the extensions resulting in higher device currents and larger heat flow through the extensions to the pads. The geometric variations from the nominal device have less of an impact on both temperature and current thus making the flared device more robust than the rectangular one.

Examining the $W_{\text {fin }}$ thermal sensitivity lines in Fig. 17(a) and in Fig. 17(b) show a thinner fin is more robust than a wider fin. For example, the thermal sensitivity of a $0.2 x$ device is larger 


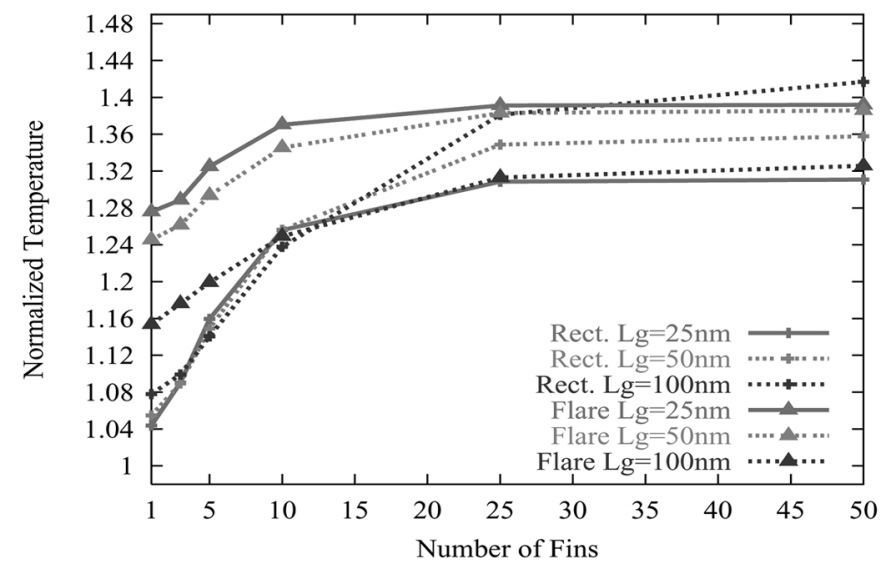

(a)

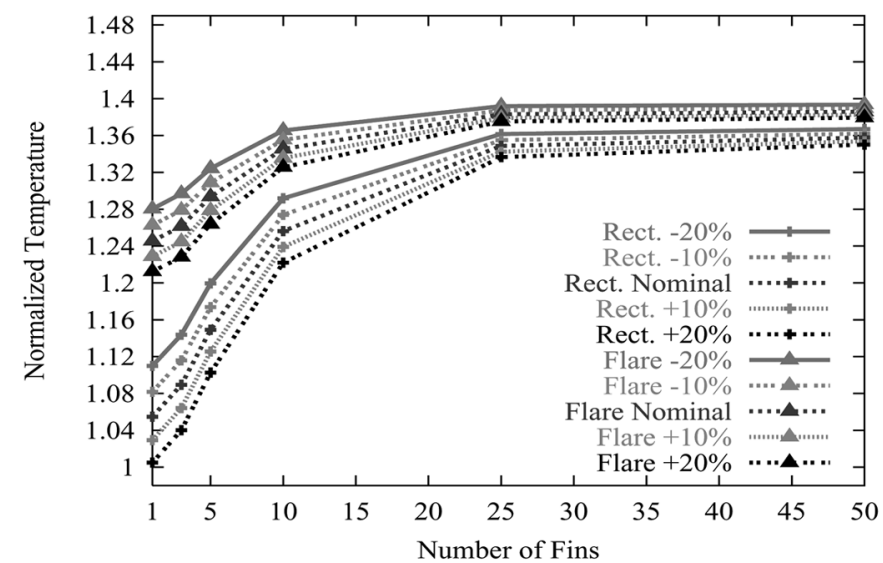

(c)

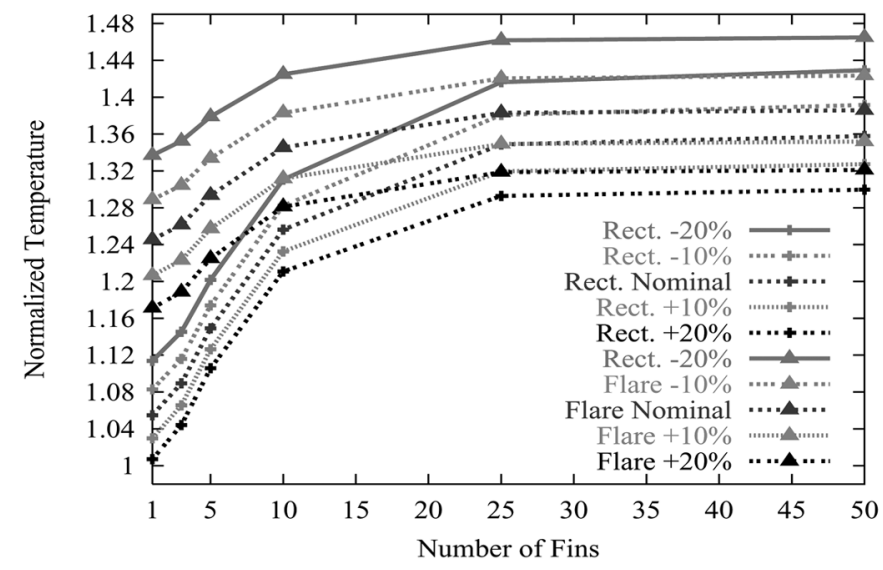

(e)

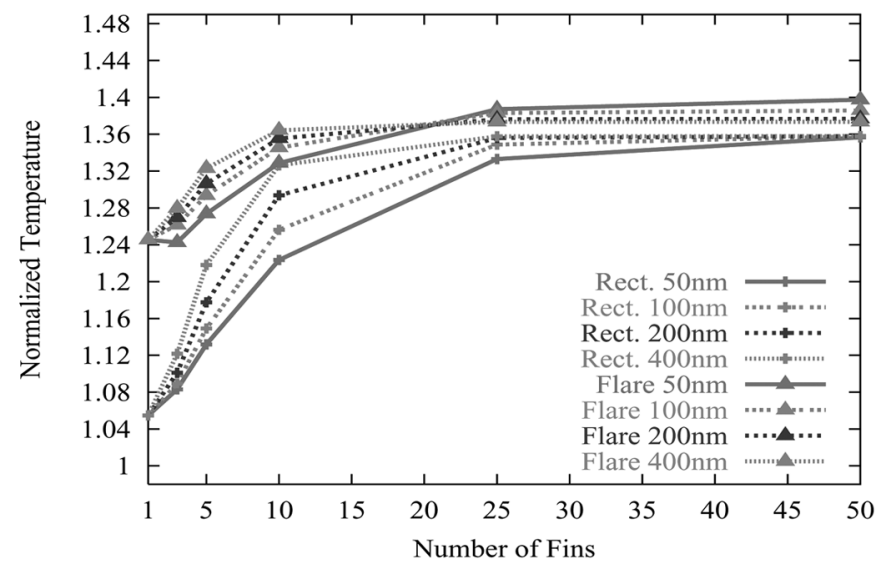

(b)

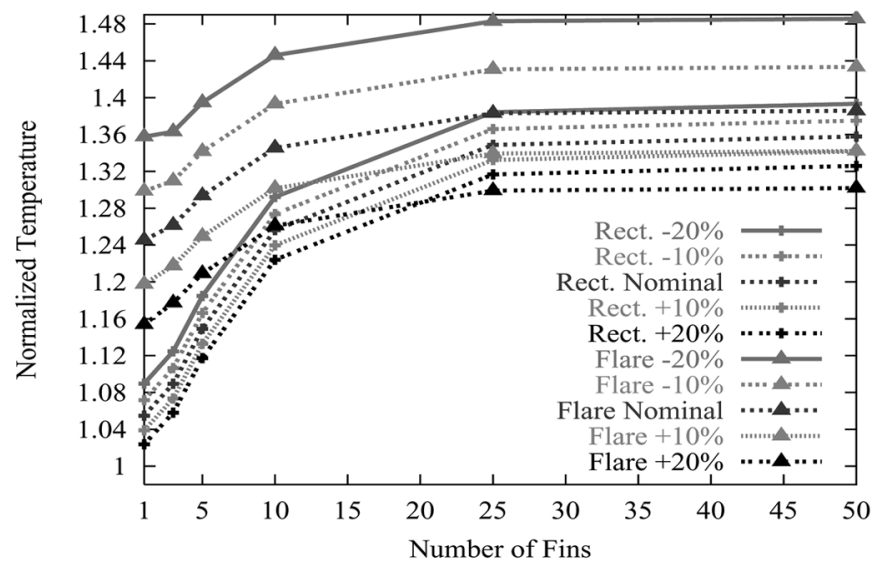

(d)

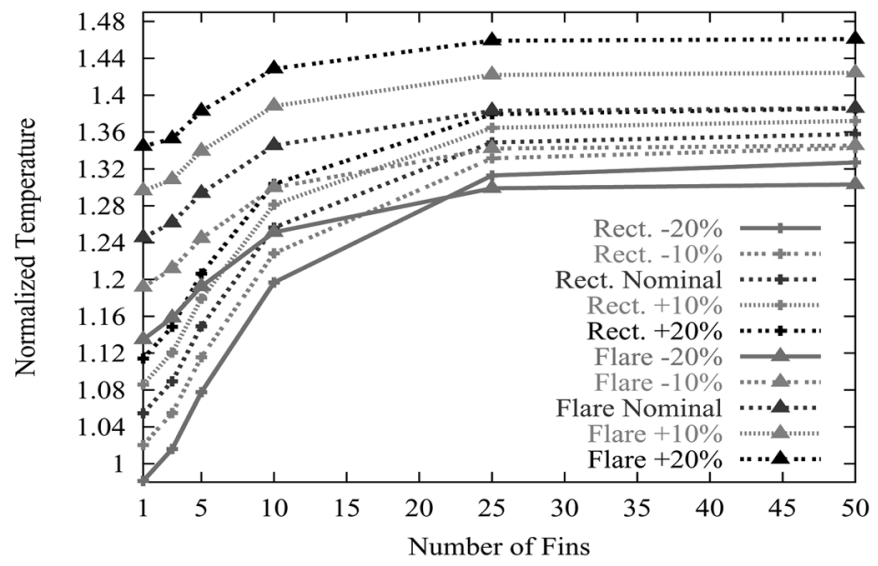

(f)

Fig. 21. Experimental data to characterize the effects of different design parameters on maximum device temperature at the drain node in rectangular channel extension devices (Rect.) and flared channel extension devices. (a) Temperature dependence on $L_{g}$. (b) Temperature dependence on $W_{\text {space }}$. (c) Temperature dependence on $H_{g}$. (d) Temperature dependence on $T_{\mathrm{ox}}$. (e) Temperature dependence on $W_{\text {fin }}$. (f) Temperature dependence on $H_{\text {fin }}$.

than the thermal sensitivity of a nominal device, thus the thinner fin is less sensitive to self-heating. This is justified when comparing the slopes of $I_{a}$ and $I_{e t}$ in Fig. 16 for the nominal and $0.2 x$ device.

From the METS plots shown in Fig. 17, some of the device variations, such as $T_{\mathrm{ox}}$ variations, contribute the least to self-heating effects. In contrast, any change in $L_{g}$ results in significant device self-heating. The circles with X's in them represent devices which do not meet recommended device geome- tries. For example, Gen et al. recommends the ratio: $W_{\text {fin }} \leq$ $0.5 L_{\mathrm{eff}}-6 t_{\mathrm{ox}}$ for reduced DIBL and ideal subthreshold slope [35]. Yu et al. provides the ratio: $W_{\text {fin }} \leq 5 L_{\text {eff }}$ for an acceptable fin aspect ratio [7]. The METS plot thus provides a good way of evaluating how the device self-heating will change under process variations. Fig. 18(a) shows the METS for a 50-fin device with rectangular channel extensions, while Fig. 18(b) shows the METS for a 50-fin with flared channel extensions. To understand the impact of geometric process variations, $W_{\text {fin }}$, 
$H_{\text {fin }}, L_{g}, H_{g}, T_{\text {ox }}$, and $W_{\text {space }}$ are varied from $0.2 x$ to $2 x$, where $1 x$ represents the nominal device (and nominal fin spacing). For each device, the METS was calculated according to (12). Similar to the single-fin devices, the 50-fin flared channel extension device exhibits higher a METS ratio than the rectangular channel extension device ( 0.81 versus 0.71$)$. It is also interesting to note the 50-fin devices are more sensitive to thermal variations than the single-fin devices, due to the larger temperature variations within the device. Fig. 18(b) shows as the fin spacing is decreased below the nominal fin spacing, the METS ratio decreases. This can be attributed to increased heat spreading within the device leading to larger temperature gradients among the fins.

The METS metric provides a numerical summary of device performance and stability over process and temperature variations. In digital applications, METS can be used to predict device stability as different device parameters are varied to meet timing/power/area constraints. In modern digital circuits, the electrical time constant (due to critical path switching speeds) typically dominates over the thermal time constant; thus allowing the use of steady-state temperatures and currents in METS calculations. METS can also be applied to devices in analog circuits. If a set of device operating points are known, METS can be calculated for each of the different regions of operation by adjusting the electrical operating point during the electro-thermal simulation, enabling device stability predictions to the different regions of operation.

\section{CONCLUSION}

We developed in this paper thermal models for single-fin flared channel extension and for multi-fin devices. We examined several factors that affect the temperatures within multi-fin devices. Fin spacing and gate height contribute to the ability to remove heat effectively away from inner fins to the gate pads. More importantly, the fin width and gate length, contribute significantly to the maximum device temperature. The number of fins clearly affect the maximum temperature where devices with fewer fins exhibit less heating. Tight thermal design constraints will limit the number of fins allowed in parallel. We have shown that coupled electro-thermal simulations are needed to accurately capture the electrical and thermal interactions within future nano scale devices. Furthermore, we have presented a thermal sensitivity metric, METS, which allows evaluating the temperature sensitivity of a device against device parameter variations.

This work is novel as it is the first to determine the key parameters needed to construct electro-thermally-aware multi-fin devices. Furthermore, our findings motivate further research into the newly emerging area of research, electro-thermal device design. There is a need to balance electrical and thermal properties. The impact of confined device geometries and ballistic electron transport on device reliability must be carefully examined. In addition, our device-level thermal study paves the way for circuit or design level thermal investigations. The multi-fin model can be directly utilized in circuit-level SPICE simulation based thermal studies to accurately represent finFET devices in a circuit. Moreover, the coupled electro-thermal simulation demonstrated at the device-level is equally applicable at the circuit-level for transistor-level simulations. Understanding thermal design sensitivities will help understand the impact of device process variations on circuit design, a critical challenge in $45 \mathrm{~nm}$ and beyond designs.

\section{ACKNOWLEDGMENT}

The authors would like to thank S. Alam for stimulating conversations and feedback during this work.

\section{REFERENCES}

[1] ITRS, "International technology roadmap for semiconductors," 2003.

[2] D. Hisamoto, W.-C. Lee, J. Kedzierski, E. Anderson, H. Takeuchi, K. Asano, T.-J. King, J. Bokor, and C. Hu, "A folded-channel MOSFET for deep-sub-tenth micron era," in Proc. Int. Electron Devices Meet., 1998, pp. 1032-1034.

[3] T. Ludwing, I. Aller, V. Gernhoefer, J. Keinert, E. Nowak, R. Joshi, A. Mueller, and S. Tomaschko, "FinFET technology for future microprocessors," in Proc. IEEE Int. SOI Conf., 2003, pp. 33-34.

[4] D. Hisamoto, W. Lee, J. Kedzierski, H. Takeuchi, K. Asano, C. Kuo, E. Anderson, T. King, J. Bokor, and C. Hu, "FinFET-A self-aligned double-gate MOSFET scaleable to $20 \mathrm{~nm}$," IEEE Trans. Electron Devices, vol. 47, no. 12, pp. 2320-2325, Dec. 2000.

[5] Y. Choi, N. Lindert, P. Xuan, S. Tang, D. Ha, E. Anderson, T. King, J. Bokor, and C. Hu, "Sub-20 nm CMOS finFET technologies," in Proc. Int. Electron Devices Meet., 2001, pp. 421-424.

[6] J. Kedzierski, D. Fried, E. Nowak, T. Kanarsky, J. Rankin, H. Hanafi, W. Natzle, D. Boyd, Y. Zhang, R. Roy, J. Newbury, C. Yu, Q. Yang, P. Saunders, C. Willets, A. Johnson, S. Cole, H. Young, N. Carpenter, D. Rakowski, B. Rainey, P. Cottrell, M. Ieong, and H.-S. Wong, "Highperformance symmetric-gate and CMOS-compatible VT asymmetricgate finFET devices," in Proc. Int. Electron Devices Meet., 2001, pp. 437-440.

[7] B. Yu, L. Chang, S. Ahmed, H. Wang, S. Bell, C.-Y. Yang, C. Tabery, C. Ho, Q. Xiang, T.-J. King, J. Bokor, and C. Hu, "FinFET scaling to $10 \mathrm{~nm}$ gate length," in Proc. Int. Electron Devices Meet., 2002, pp. 252-254.

[8] F. Yang, H. Chen, F. Chen, Y. Chan, K. Yang, C. Chen, H. Tao, Y. C. Adn, M. Liang, and C. Hu, "35 nm CMOS finFETs," in Proc. Symp. VLSI Technol., 2002, pp. 104-105.

[9] K. Jenkins and R. Franch, "Impact of self-heating on digital SOI and strained-silicon CMOS circuits," in Proc. IEEE Int. SOI Conf., 2003, pp. 161-163.

[10] R. Joy and E. Schling, "Thermal properties of very fast transistors," IEEE Trans. Electron Devices, vol. 17, no. 4, pp. 586-594, Aug. 1970.

[11] N. Rinaldi, "On the modeling of the transient thermal behavior of semiconductor devices," IEEE Trans. Electron Devices, vol. 48, no. 12, pp. 2796-2802, Dec. 2001.

[12] P. Sverdrup, Y. Ju, and K. Goodson, "Sub-continuum simulations of heat conduction in silicon-on-Insulator transistors," J. Heat Transfer, vol. 123, no. 1, pp. 130-137, Feb. 2001.

[13] E. Pop, K. Banerjee, P. Sverdrup, and K. Goodson, "Localized heating effects and scaling of sub-0.18 micron CMOS devices," in Proc. Int. Electron Devices Meet., Dec. 2001, pp. 677-680.

[14] Y. Ju and K. Goodson, "Phonon scattering in silicon films of thickness below 100 nm," Appl. Phys. Lett., vol. 74, pp. 3005-3007, 1999.

[15] E. Pop, R. Dutton, and K. Goodson, "Thermal analysis of ultra-thin body device scaling," in Proc. Int. Electron Devices Meet., 2003, pp. 36.6.1-36.6.4.

[16] L. Su, J. Chung, D. Antoniadis, K. Goodson, and M. Flik, "Measurement and modeling of self-heating in SOI nMOSFETs," IEEE Trans. Electron Devices, vol. 41, no. 1, pp. 69-75, Jan. 1994.

[17] S. Tang, L. Chang, N. Lindert, Y. Choi, W. Lee, X. Huang, V. Subramanian, J. Bokor, T. King, and C. Hu, "FinFET - a quasi-planar double gate MOSFET," in Proc. IEEE Int. Solid-State Circuits Conf., 2001, pp. $118-119$.

[18] K. Bernstein, C. Chaung, R. Joshi, and R. Puri, "Design and CAD challenges in sub-90 nm CMOS technologies," in Proc. Int. Conf. Comput.Aided Des., 2003, pp. 129-136.

[19] E. Pop and K. Goodson, "Thermal phenomena in nanoscale transistors," in Proc. Intersoc. Conf. Thermal Thermomechan. Phenomena Electronic Syst., 2004, pp. 1-7.

[20] U. Lindefelt, "Heat generation in semiconductor devices," J. Appl. Phys., vol. 75, no. 2, pp. 942-957, Jan. 1994. 
[21] C. Teng, Y. Cheng, E. Rosenhaum, and S. Kang, "ITEM: A temperature dependent electromigration reliability diagnosis tool," IEEE Trans. Comput.-Aided Des. Integr. Circuits Syst., vol. 16, no. 8, pp. 882-893, Aug. 1997.

[22] J. Kedzierski, M. Ieong, E. Nowak, T. Kanarsky, Y. Zhang, R. Roy, D. Boyd, D. Fried, and H.-S. Wong, "Extension and source/drain design for high-performance FinFET devices," IEEE Trans. Electron Devices, vol. 50, no. 4, pp. 952-958, Apr. 2003.

[23] B. Swahn, "FinFETs: Thermal modeling, analysis, and circuit design," Ph.D. dissertation, Dept. Elect. Comput. Eng., Tufts University, Somerville, MA, 2006.

[24] M. Lundstrom and Z. Ren, "Essential physics of carrier transport in nanoscale MOSFETs," IEEE Trans. Electron Devices, vol. 49, no. 1, pp. 133-141, Jan. 2002.

[25] B. Krabbenborg, A. Bosma, H. de Graaff, and A. Mouthaan, "Layout to circuit extraction for three-dimensional thermal-electrical circuit simulation of device structures," IEEE Trans. Comput.-Aided Des. Integr. Circuits Syst., vol. 15, no. 7, pp. 765-774, Jul. 1996.

[26] G. Digele, S. Lindenkreuz, and E. Kasper, "Fully coupled dynamic electro-thermal simulation," IEEE Trans. Very Large Scale Integr. (VLSI) Syst., vol. 5, no. 3, pp. 250-257, Sep. 1997.

[27] V. Székely, A. Poppe, A. Pahi, A. Csendes, G. Hajas, and M. Rencz, "Electro-thermal and logi-thermal simulation of VLSI designs," IEEE Trans. Very Large Scale Integr. (VLSI) Syst., vol. 5, no. 3, pp. 258-269, Sep. 1997.

[28] M. Rencz, V. Székely, and A. Poppe, "A fast algorithm for the layout based electro-thermal simulation," in Proc. Des., Autom. Test Eur. Conf., 2003, pp. 1032-1037.

[29] L. Codecasa, D. D'Amore, and P. Maffezzoni, "Compact modeling of electrical devices for electrothermal analysis," IEEE Trans. Circuits Syst. I, Fundam. Theory Appl., vol. 50, no. 4, pp. 465-476, Apr. 2003.

[30] Y. Zhan and S. Sapatnekar, "Fast computation of the temperature distribution in VLSI chips using the discrete cosine transform and table look-up," in Proc. Asia South Pacific Des. Autom. Conf., 2005, pp. 87-92.

[31] C. Liu, E. Carlen, K. Wise, and C. Mastrangelo, "Simulation of electrothermal MOS circuits using saber," in Proc. Int. Conf. Model. Simulation Microsyst., Semiconduct., Sensors Actuators, 1998, pp. 239-244.

[32] T.-Y. Chiang, K. Banerjee, and K. Saraswat, "Compact modeling and SPICE-based simulation for electrothermal analysis of multilevel ulsi interconnects," in Proc. Int. Conf. Comput.-Aided Des., 2001, pp. $165-172$.

[33] C.-H. Lin, M. Chan, X. Xi, J. He, X. Liang, W. Wang, Y. Taur, A. Niknejad, and C. Hu, BSIMDG 1.0 Double-Gate MOSFET Model Technology Note (Alpha-Release). Berkeley, CA: Department of Electrical Engineering and Computer Sciences, University of California, 2003
[34] B. Swahn and S. Hassoun, "METS: A Metric for Electro-Thermal Sensitivity, and its application to FinFETs," in Proc. Int. Symp. Quality Electron. Des., 2006, pp. 121-126.

[35] G. Pei, V. Narayanan, Z. Liu, and E. Kan, "3-D analytical subthreshold and quantum mechanical analysis of double-gate MOSFET," in Proc. Int. Electron Devices Meet., Dec. 2001, pp. 103-106.

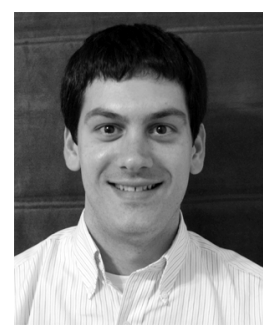

Brian Swahn (M'07) received the B.S. degree in electrical engineering and the B.S. degree in computer engineering from The Pennsylvania State University, Erie, in 2001, and the M.S. and Ph.D. degrees in electrical engineering from Tufts University, Somerville, MA, in 2002 and 2006, respectively.

He is currently with Analog Devices, Inc., Wilmington, MA. His research interests include analog and mixed-signal simulation, parasitic extraction, device modeling, device and interconnect reliability, timing analysis, and circuit optimization.

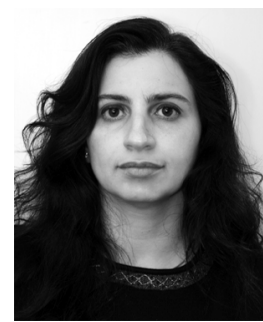

Soha Hassoun (SM'07) received the M.S. degree from Massachusetts Institute of Technology, Cambridge, in 1988, and the Ph.D. degree from the University of Washington, Seattle, in 1997.

Currently, she is an Associate Professor with the Department of Computer Science, Tufts University, Medford, MA. From 1988 to 1991, she worked with Digital Equipment Corporation, Hudson, MA. She worked with IBM Research Labs, Austin, TX, in 2002. She worked for Carbon Design Systems in 2007. Her research interests include synthesis, timing and thermal analysis, and physical design.

Dr. Hassoun served as ICCAD's technical program chair in 2005 and as general chair in 2006. He serves on the IEEE Council on EDA and has served on the advisory board for ACM's Special Interest Group on Design Automation (SIGDA). He was an Associate Editor for the IEEE TRANSACTIONS ON COMPUTER-AIDED DESIGN OF INTEGRATED CiRCUITS AND SySTEMS and currently serves as an Editor for the IEEE Design and Test Magazine. She was a recipient of the NSF CAREER Award and, in June 2007, she received the ACM/ SIGDA Distinguished Service Award for creating the Ph.D. forum at DAC. 